\title{
The stability analysis of an epidemic model with saturating incidence and age-structure in the exposed and infectious classes
}

\author{
Yuji Li ${ }^{1}$, Rui $X u^{2,3^{*}}$ and Jiazhe Lin ${ }^{1}$
}

"Correspondence: rxu88@163.com ${ }^{2}$ Complex Systems Research Center, Shanxi University, Taiyuan, P.R. China ${ }^{3}$ Shanxi Key Laboratory of Mathematical Techniques and Big Data Analysis on Disease Control and Prevention, Shanxi University, Taiyuan, P.R. China

Full list of author information is available at the end of the article

\section{Springer}

\begin{abstract}
In this paper, an HBV epidemic model that incorporates saturating incidence and age-structure in the exposed and infectious classes is proposed. We study the asymptotic smoothness of semi-flow generated by the model. By calculating the basic reproduction number and analyzing the characteristic equations, the local stability of disease-free and endemic steady states is studied. We investigate the global dynamics of this model by using Lyapunov functionals and LaSalle's invariance principle and prove that, if the basic reproduction number is less than unity, the disease-free steady state is globally asymptotically stable; if the basic reproduction number is greater than unity, the endemic steady state is globally asymptotically stable.
\end{abstract}

Keywords: Age-structured model; Saturating incidence; Asymptotic smoothness; Lyapunov functional; Global stability

\section{Introduction}

Hepatitis B is very contagious and it is hard to control its transmission. Thus studying the law of its infection has become the focus of attention. Researchers have presented an HBV epidemic model to study hepatitis B transmission and some results of the investigation have been given. The model's dynamics is determined by the basic reproduction number (the average number of secondary infections caused by one infectious individual in its duration of infection). When the basic reproduction number is less than unity, the diseasefree steady state is globally asymptotically stable and all hepatitis B patients will recover; when the basic reproduction number is larger than unity, there exists a unique endemic steady state and it is globally asymptotically stable.

Although there exist many epidemic models, some are not appropriate to show the hepatitis B transmission rules. In [1], Liu divided hepatitis B patients into acute and chronic patients and presented an ordinary differential model. However, the scaled probability of hepatitis $B$ virus infection is connected with the age of infection and the risk per unit time of activation appears to be higher in the early stages of infection than in later stages.

(c) The Author(s) 2018. This article is distributed under the terms of the Creative Commons Attribution 4.0 International License (http://creativecommons.org/licenses/by/4.0/), which permits unrestricted use, distribution, and reproduction in any medium, provided you give appropriate credit to the original author(s) and the source, provide a link to the Creative Commons license, and indicate if changes were made. 
Therefore, in [2], Li presented the following model:

$$
\begin{aligned}
& \dot{S}(t)=\Lambda-(\mu+p) S(t)-S(t) \int_{0}^{\infty} \beta_{1}(a) i(t, a) \mathrm{d} a-S(t) \int_{0}^{\infty} \beta_{3}(a) j(t, a) \mathrm{d} a, \\
& \dot{V}(t)=p S(t)-(\mu+\rho) V(t)-V(t) \int_{0}^{\infty} \beta_{2}(a) i(t, a) \mathrm{d} a \\
& -V(t) \int_{0}^{\infty} \beta_{4}(a) j(t, a) \mathrm{d} a, \\
& \frac{\partial e(t, a)}{\partial a}+\frac{\partial e(t, a)}{\partial t}=-\theta_{1}(a) e(t, a) \\
& \frac{\partial i(t, a)}{\partial a}+\frac{\partial i(t, a)}{\partial t}=-\theta_{2}(a) i(t, a), \\
& \frac{\partial j(t, a)}{\partial a}+\frac{\partial j(t, a)}{\partial t}=-\theta_{3}(a) j(t, a), \\
& \dot{R}(t)=\rho V(t)+\int_{0}^{\infty} \delta_{1}(a) i(t, a) \mathrm{d} a+\int_{0}^{\infty} \delta_{2}(a) j(t, a) \mathrm{d} a-\mu R(t),
\end{aligned}
$$

with boundary conditions

$$
\begin{aligned}
e(t, 0)= & S(t) \int_{0}^{\infty}\left(\beta_{1}(a) i(t, a)+\beta_{3}(a) j(t, a)\right) \mathrm{d} a \\
& +V(t) \int_{0}^{\infty}\left(\beta_{2}(a) i(t, a)+\beta_{4}(a) j(t, a)\right) \mathrm{d} a, \\
i(t, 0)= & \int_{0}^{\infty} \gamma_{1}(a) e(t, a) \mathrm{d} a, \\
j(t, 0)= & \int_{0}^{\infty} \gamma_{2}(a) e(t, a) \mathrm{d} a+\int_{0}^{\infty} \xi(a) i(t, a) \mathrm{d} a .
\end{aligned}
$$

It seems that vaccination has been the most effective prevention measure against hepatitis B. However, clinic evidence shows that some vaccines gradually lost immunity soon after vaccination. In other words, even though all individuals have been vaccinated, many of them may still be infected. Therefore, it is necessary to consider that a small part of vaccines will be susceptible individuals after a period of time. Furthermore, acute hepatitis $B$ patients are highly infectious and they have apparent symptoms, the government and hospital have to take strategies as soon as possible, such like quarantine measures, therapeutic measures and so on. Considering the real transmission rules of hepatitis B, it is necessary to introduce saturating incidence to describe the law of hepatitis B's infection. Therefore, we propose an epidemic model as follows:

$$
\begin{aligned}
& \dot{S}(t)=\Lambda-(\mu+p) S(t)+\eta V(t)-S(t) \int_{0}^{\infty}\left(\frac{\beta_{1}(a) i(t, a)}{1+\alpha i(t, a)}+\beta_{3}(a) j(t, a)\right) \mathrm{d} a, \\
& \dot{V}(t)=p S(t)-(\mu+\rho+\eta) V(t)-V(t) \int_{0}^{\infty}\left(\frac{\beta_{2}(a) i(t, a)}{1+\alpha i(t, a)}+\beta_{4}(a) j(t, a)\right) \mathrm{d} a, \\
& \frac{\partial e(t, a)}{\partial a}+\frac{\partial e(t, a)}{\partial t}=-\theta_{1}(a) e(t, a), \\
& \frac{\partial i(t, a)}{\partial a}+\frac{\partial i(t, a)}{\partial t}=-\theta_{2}(a) i(t, a),
\end{aligned}
$$




$$
\begin{aligned}
& \frac{\partial j(t, a)}{\partial a}+\frac{\partial j(t, a)}{\partial t}=-\theta_{3}(a) j(t, a), \\
& \dot{R}(t)=\rho V(t)+\int_{0}^{\infty} \delta_{1}(a) i(t, a) \mathrm{d} a+\int_{0}^{\infty} \delta_{2}(a) j(t, a) \mathrm{d} a-\mu R(t),
\end{aligned}
$$

where

$$
\begin{aligned}
& \theta_{1}(a)=\mu+\gamma_{1}(a)+\gamma_{2}(a), \quad \theta_{2}(a)=\mu+\delta_{1}(a)+\varepsilon_{1}(a)+\xi(a), \\
& \theta_{3}(a)=\mu+\delta_{2}(a)+\varepsilon_{2}(a)
\end{aligned}
$$

with boundary conditions

$$
\begin{aligned}
e(t, 0)= & S(t) \int_{0}^{\infty}\left(\frac{\beta_{1}(a) i(t, a)}{1+\alpha i(t, a)}+\beta_{3}(a) j(t, a)\right) \mathrm{d} a \\
& +V(t) \int_{0}^{\infty}\left(\frac{\beta_{2}(a) i(t, a)}{1+\alpha i(t, a)}+\beta_{4}(a) j(t, a)\right) \mathrm{d} a, \\
i(t, 0)= & \int_{0}^{\infty} \gamma_{1}(a) e(t, a) \mathrm{d} a, \\
j(t, 0)= & \int_{0}^{\infty} \gamma_{2}(a) e(t, a) \mathrm{d} a+\int_{0}^{\infty} \xi(a) i(t, a) \mathrm{d} a,
\end{aligned}
$$

and initial conditions

$$
\begin{aligned}
& S(0)=\varphi_{S} \geq 0, \quad V(0)=\varphi_{V} \geq 0, \quad e(0, a)=\varphi_{e}(a) \in L_{+}^{1}(0, \infty), \\
& i(0, a)=\varphi_{i}(a) \in L_{+}^{1}(0, \infty), \quad j(0, a)=\varphi_{j}(a) \in L_{+}^{1}(0, \infty), \quad R(0)=R_{0} \geq 0,
\end{aligned}
$$

where $e(t, a)$ represents the density of exposed individuals with age of latency $a$ at time $t$. $i(t, a), j(t, a)$ represent the density of patients with acute hepatitis B and chronic hepatitis B with age of infection $a$ at time $t$, respectively. $\alpha$ means the saturating incidence coefficient. The parameters of model (1.2) are biologically explained in Table 1.

Table 1 Parameters and their biological meaning in model (1.2)

\begin{tabular}{ll}
\hline Parameter & Interpretation \\
\hline$\Lambda$ & constant recruitment rate \\
$\mu$ & natural death rate \\
$p$ & the rate for susceptible individuals to be vaccinated \\
$\rho$ & the rate for vaccinees to obtain immunity and move into recovered population \\
$\eta$ & the proportion of vaccines that lose efficacy \\
$\beta_{1}(a)$ & the rate for acute hepatitis B patients infecting susceptible individuals at age $a$ \\
$\beta_{2}(a)$ & the rate for acute hepatitis B patients infecting vaccinees at age $a$ \\
$\beta_{3}(a)$ & the rate for chronic hepatitis B patients infecting susceptible individuals at age $a$ \\
$\beta_{4}(a)$ & the rate for chronic hepatitis B patients infecting vaccinees at age $a$ \\
$\gamma_{1}(a)$ & the rate for exposed individuals being acute hepatitis B patients at age $a$ \\
$\gamma_{2}(a)$ & the rate for exposed individuals being chronic hepatitis B patients at age $a$ \\
$\varepsilon_{1}(a)$ & acute hepatitis B death rate at age $a$ \\
$\varepsilon_{2}(a)$ & chronic hepatitis B death rate at age $a$ \\
$\delta_{1}(a)$ & the rate for acute hepatitis B patients being recovered population at age $a$ \\
$\delta_{2}(a)$ & the rate for chronic hepatitis B patients being recovered population at age $a$ \\
\hline
\end{tabular}


Since the variable $R(t)$ does not appear in the first five equations of (1.2), in this paper, we consider the following subsystem:

$$
\begin{aligned}
& \dot{S}(t)=\Lambda-(\mu+p) S(t)+\eta V(t)-S(t) \int_{0}^{\infty}\left(\frac{\beta_{1}(a) i(t, a)}{1+\alpha i(t, a)}+\beta_{3}(a) j(t, a)\right) \mathrm{d} a, \\
& \dot{V}(t)=p S(t)-(\mu+\rho+\eta) V(t)-V(t) \int_{0}^{\infty}\left(\frac{\beta_{2}(a) i(t, a)}{1+\alpha i(t, a)}+\beta_{4}(a) j(t, a)\right) \mathrm{d} a, \\
& \frac{\partial e(t, a)}{\partial a}+\frac{\partial e(t, a)}{\partial t}=-\theta_{1}(a) e(t, a), \\
& \frac{\partial i(t, a)}{\partial a}+\frac{\partial i(t, a)}{\partial t}=-\theta_{2}(a) i(t, a), \\
& \frac{\partial j(t, a)}{\partial a}+\frac{\partial j(t, a)}{\partial t}=-\theta_{3}(a) j(t, a), \\
& e(t, 0)=S(t) \int_{0}^{\infty}\left(\frac{\beta_{1}(a) i(t, a)}{1+\alpha i(t, a)}+\beta_{3}(a) j(t, a)\right) \mathrm{d} a \\
& \quad+V(t) \int_{0}^{\infty}\left(\frac{\beta_{2}(a) i(t, a)}{1+\alpha i(t, a)}+\beta_{4}(a) j(t, a)\right) \mathrm{d} a, \\
& i(t, 0)=\int_{0}^{\infty} \gamma_{1}(a) e(t, a) \mathrm{d} a, \\
& j(t, 0)=\int_{0}^{\infty} \gamma_{2}(a) e(t, a) \mathrm{d} a+\int_{0}^{\infty} \xi(a) i(t, a) \mathrm{d} a .
\end{aligned}
$$

This paper is organized as follows. In Sect. 2, we introduce some basic results of system (1.5), including state space, assumptions and boundedness of the solutions. Asymptotic smoothness of the semi-flow is analyzed in Sect. 3, which is generated by the system (1.5). Then we study the existence of equilibria and obtain the expression of the basic reproduction number $R_{0}$ in Sect. 4 . The local stability of equilibria is proved in Sect. 5, while the uniform persistence of the system (1.5) is verified in Sect. 6. In Sect. 7, we give a proof of the global stability of equilibria. A brief remark is given in Sect. 8 to conclude this work.

More details concerning the global stability analysis of epidemic model, we refer the reader to [3-23].

\section{Preliminaries}

To make the model be biologically significant, we list the assumption as follows:

Assumption 1 We assume that

(i) $\beta_{1}(a), \beta_{2}(a), \beta_{3}(a), \beta_{4}(a), \theta_{1}(a), \theta_{2}(a), \xi(a)$ are non-negative and belong to $L_{+}^{\infty}(0, \infty)$ with respective essential upper bound $\bar{\beta}_{1}, \bar{\beta}_{2}, \bar{\beta}_{3}, \bar{\beta}_{4}, \bar{\theta}_{1}, \bar{\theta}_{2}, \bar{\xi} \in(0, \infty)$;

(ii) $\beta_{1}(a), \beta_{2}(a), \beta_{3}(a), \beta_{4}(a), \xi(a)$ are Lipschitz continuous on $R_{+}$with coefficients $M_{\beta_{1}}, M_{\beta_{2}}, M_{\beta_{3}}, M_{\beta_{4}}, M_{\xi}$, respectively;

(iii) there exists a positive constant $\mu_{0} \in(0, \mu]$ such that $\left|\theta_{1}(a)-\gamma_{1}(a)-\gamma_{2}(a)\right| \geq \mu_{0}$, $\left|\theta_{2}(a)-\xi(a)\right| \geq \mu_{0}, \theta_{3}(a) \geq \mu_{0}$, for all $a>0$.

\subsection{State space}

Define the space of functions $X$ as

$$
X=R_{+} \times R_{+} \times L_{+}^{1}(0, \infty) \times L_{+}^{1}(0, \infty) \times L_{+}^{1}(0, \infty),
$$


equipped with the norm

$$
\left\|\left(x_{1}, x_{2}, x_{3}, x_{4}, x_{5}\right)\right\|_{\mathcal{X}}=\left|x_{1}\right|+\left|x_{2}\right|+\int_{0}^{\infty}\left|x_{3}(a)\right| \mathrm{d} a+\int_{0}^{\infty}\left|x_{4}(a)\right| \mathrm{d} a+\int_{0}^{\infty}\left|x_{5}(a)\right| \mathrm{d} a .
$$

Then the initial values (1.4) of system (1.5) are taken to be included in $X$ :

$$
(S(0), V(0), e(0, a), i(0, a), j(0, a))=\left(S_{0}, V_{0}, \varphi_{e}(a), \varphi_{i}(a), \varphi_{j}(a)\right) \in X .
$$

By the standard theory of functional differential equations [24], it can be verified that system (1.5) with initial conditions (1.4) has a unique non-negative solution. Thus, we have a continuous semi-flow associated with system (1.5), that is,

$$
\Phi_{t}\left(X_{0}\right):=X(t)=(S(t), V(t), e(t, \cdot), i(t, \cdot), j(t, \cdot)) \in \mathcal{X}, \quad t \geq 0,
$$

with

$$
\begin{aligned}
\left\|\Phi_{t}\left(X_{0}\right)\right\|_{\mathcal{X}} & =\|S(t), V(t), e(t, \cdot), i(t, \cdot), j(t, \cdot)\|_{\mathcal{X}} \\
& =|S(t)|+|V(t)|+\int_{0}^{\infty}|e(t, a)| \mathrm{d} a+\int_{0}^{\infty}|i(t, a)| \mathrm{d} a+\int_{0}^{\infty}|j(t, a)| \mathrm{d} a .
\end{aligned}
$$

Finally, we define the state space for system (1.5) as

$$
\begin{aligned}
\Upsilon:= & \left\{(S(t), V(t), e(t, \cdot), i(t, \cdot), j(t, \cdot)) \in \mathcal{X}: 0 \leq S(t)+V(t)+\int_{0}^{\infty} e(t, a) \mathrm{d} a\right. \\
& \left.+\int_{0}^{\infty} i(t, a) \mathrm{d} a+\int_{0}^{\infty} j(t, a) \mathrm{d} a \leq \frac{\Lambda}{\mu_{0}}\right\},
\end{aligned}
$$

which can be proved to be positive invariant by the following proposition.

\subsection{Boundedness}

The last three equations of system (1.5) can be reformulated as Volterra equations by use of Volterra formulation. In order to be convenient for computation, we denote

$$
\begin{aligned}
& B_{1}(a)=\exp \left(-\int_{0}^{a} \theta_{1}(\tau) \mathrm{d} \tau\right), \quad B_{2}(a)=\exp \left(-\int_{0}^{a} \theta_{2}(\tau) \mathrm{d} \tau\right), \\
& B_{3}(a)=\exp \left(-\int_{0}^{a} \theta_{3}(\tau) \mathrm{d} \tau\right) .
\end{aligned}
$$

From the expressions of $B_{1}(a), B_{2}(a)$ and $B_{3}(a)$, according to Assumption 1, it is easy to see that, for all $a \geq 0$,

$$
\begin{aligned}
& 0 \leq B_{1}(a), B_{2}(a), B_{3}(a) \leq e^{-\mu_{0} a}, \\
& B_{1}^{\prime}(a)=-\theta_{1}(a) B_{1}(a), \quad B_{2}^{\prime}(a)=-\theta_{2}(a) B_{2}(a), \quad B_{3}^{\prime}(a)=-\theta_{3}(a) B_{3}(a) .
\end{aligned}
$$


By integrating the terms $e(t, a), i(t, a)$ and $j(t, a)$ along the characteristic line $t-a=$ const., respectively, we get the following expressions:

$$
\begin{aligned}
& e(t, a)= \begin{cases}e(t-a, 0) B_{1}(a) & \text { for } 0 \leq a \leq t, \\
\varphi_{e}(a-t) \frac{B_{1}(a)}{B_{1}(a-t)} & \text { for } 0 \leq t \leq a,\end{cases} \\
& i(t, a)= \begin{cases}i(t-a, 0) B_{2}(a) & \text { for } 0 \leq a \leq t, \\
\varphi_{i}(a-t) \frac{B_{2}(a)}{B_{2}(a-t)} & \text { for } 0 \leq t \leq a,\end{cases} \\
& j(t, a)= \begin{cases}j(t-a, 0) B_{3}(a) & \text { for } 0 \leq a \leq t, \\
\varphi_{j}(a-t) \frac{B_{3}(a)}{B_{3}(a-t)} & \text { for } 0 \leq t \leq a .\end{cases}
\end{aligned}
$$

Before analyzing the boundedness of system (1.5), we first show the non-negative of the solution. From (2.2)-(2.4), it is not difficult to verify that $e(t, a), i(t, a)$ and $j(t, a)$ are nonnegative, due to the non-negativity of $B_{1}(a), B_{2}(a), B_{3}(a)$, and the non-negative initial conditions (1.4). From the first equation of (1.3), we see that $S(t)>0$ holds or $V(t)>0$ holds. For the sake of contradiction, if $V(t)>0$ and $S(t) \leq 0$, from the second equation of (1.5), we know that $\dot{V}(t)<0$. Then $V(t)$ is a monotone decreasing function with respect to $t$ and there exists at least one zero solution, which contradicts $V(t)>0$. If $S(t)>0$ and $V(t) \leq 0, V(t)$ is a monotone increasing function with respect to $t$, which contradicts the later boundedness analysis. Thus, all the solutions of system (1.5) remain non-negative.

In order to imply the boundedness of system (1.5), we have the following proposition.

Proposition 2.1 Consider system (1.5) and Eq. (2.1), we have

(i) $\Upsilon$ is positively invariant for $\Phi_{t}$, that is, $\Phi_{t}\left(X_{0}\right) \in \Upsilon$, for $\forall t \geq 0, X_{0} \in \Upsilon$;

(ii) $\Phi_{t}$ is point dissipative: there is a bounded set that attracts all points in $\mathcal{X}$.

Proof Note that

$$
\begin{aligned}
\frac{\mathrm{d}}{\mathrm{d} t}\left\|\Phi_{t}\left(X_{0}\right)\right\|_{\mathcal{X}}= & \frac{\mathrm{d} S(t)}{\mathrm{d} t}+\frac{\mathrm{d} V(t)}{\mathrm{d} t}+\frac{\mathrm{d}}{\mathrm{d} t} \int_{0}^{\infty} e(t, a) \mathrm{d} a \\
& +\frac{\mathrm{d}}{\mathrm{d} t} \int_{0}^{\infty} i(t, a) \mathrm{d} a+\frac{\mathrm{d}}{\mathrm{d} t} \int_{0}^{\infty} j(t, a) \mathrm{d} a .
\end{aligned}
$$

By Eq. (2.2), we get

$$
\int_{0}^{\infty} e(t, a) \mathrm{d} a=\int_{0}^{t} e(t-a, 0) B_{1}(a) \mathrm{d} a+\int_{t}^{\infty} \varphi_{e}(a-t) \frac{B_{1}(a)}{B_{1}(a-t)} \mathrm{d} a
$$

Taking the substitution $\sigma=t-a$ and $\tau=a-t$ in the first and second integral, respectively, and differentiating by $t$, we get

$$
\begin{aligned}
\frac{\mathrm{d}}{\mathrm{d} t} \int_{0}^{\infty} e(t, a) \mathrm{d} a & =\frac{\mathrm{d}}{\mathrm{d} t} \int_{0}^{t} e(\sigma, 0) B_{1}(t-\sigma) \mathrm{d} \sigma+\frac{\mathrm{d}}{\mathrm{d} t} \int_{0}^{\infty} \varphi_{e}(\tau) \frac{B_{1}(t+\tau)}{B_{1}(\tau)} \mathrm{d} \tau \\
& =e(t, 0) B_{1}(0)+\int_{0}^{\infty} \varphi_{e}(\tau) \frac{B_{1}^{\prime}(t+\tau)}{B_{1}(\tau)} \mathrm{d} \tau+\int_{0}^{t} e(\sigma-a) B_{1}^{\prime}(t-\sigma) \mathrm{d} \sigma
\end{aligned}
$$


Noting that $B_{1}(0)=1$ and $B_{1}^{\prime}(a)=-\theta_{1}(a) B_{1}(a)$, we obtain

$$
\frac{\mathrm{d}}{\mathrm{d} t} \int_{0}^{\infty} e(t, a) \mathrm{d} a=e(t, 0)-\int_{0}^{\infty} \theta_{1}(a) e(t, a) \mathrm{d} a .
$$

Similarly, we have

$$
\begin{aligned}
& \frac{\mathrm{d}}{\mathrm{d} t} \int_{0}^{\infty} i(t, a) \mathrm{d} a=i(t, 0)-\int_{0}^{\infty} \theta_{2}(a) i(t, a) \mathrm{d} a \\
& \frac{\mathrm{d}}{\mathrm{d} t} \int_{0}^{\infty} j(t, a) \mathrm{d} a=j(t, 0)-\int_{0}^{\infty} \theta_{3}(a) j(t, a) \mathrm{d} a .
\end{aligned}
$$

By (2.6), (2.7) and (2.8), Eq. (2.5) becomes

$$
\begin{aligned}
\frac{\mathrm{d}}{\mathrm{d} t}\left\|\Phi_{t}\left(X_{0}\right)\right\|_{\mathcal{X}}= & \Lambda-\mu S(t)-(\mu+\rho) V(t)-S(t) \int_{0}^{\infty}\left(\frac{\beta_{1}(a) i(t, a)}{1+\alpha i(t, a)}+\beta_{3}(a) j(t, a)\right) \mathrm{d} a \\
& -V(t) \int_{0}^{\infty}\left(\frac{\beta_{2}(a) i(t, a)}{1+\alpha i(t, a)}+\beta_{4}(a) j(t, a)\right) \mathrm{d} a \\
& +S(t) \int_{0}^{\infty}\left(\frac{\beta_{1}(a) i(t, a)}{1+\alpha i(t, a)}+\beta_{3}(a) j(t, a)\right) \mathrm{d} a \\
& +V(t) \int_{0}^{\infty}\left(\frac{\beta_{2}(a) i(t, a)}{1+\alpha i(t, a)}+\beta_{4}(a) j(t, a)\right) \mathrm{d} a \\
& -\int_{0}^{\infty} \theta_{1}(a) e(t, a) \mathrm{d} a+\int_{0}^{\infty} \gamma_{1}(a) e(t, a) \mathrm{d} a-\int_{0}^{\infty} \theta_{2}(a) i(t, a) \mathrm{d} a \\
& +\int_{0}^{\infty} \gamma_{2}(a) e(t, a) \mathrm{d} a+\int_{0}^{\infty} \xi(a) i(t, a) \mathrm{d} a-\int_{0}^{\infty} \theta_{3}(a) j(t, a) \mathrm{d} a \\
= & \Lambda-\mu S(t)-(\mu+\rho) V(t)-\int_{0}^{\infty}\left(\theta_{1}(a)-\gamma_{1}(a)-\gamma_{2}(a)\right) e(t, a) \mathrm{d} a \\
& -\int_{0}^{\infty}\left(\theta_{2}(a)-\xi(a)\right) i(t, a) \mathrm{d} a-\int_{0}^{\infty} \theta_{3}(a) j(t, a) \mathrm{d} a .
\end{aligned}
$$

Thus, from (iii) of Assumption 1, we can get

$$
\begin{aligned}
\frac{d}{d t}\left\|\Phi_{t}\left(X_{0}\right)\right\|_{\mathcal{X}} \leq & \Lambda-\mu S(t)-(\mu+\rho) V(t) \\
& -\mu_{0}\left(\int_{0}^{\infty} e(t, a) \mathrm{d} a+\int_{0}^{\infty} i(t, a) \mathrm{d} a+\int_{0}^{\infty} j(t, a) \mathrm{d} a\right) \\
\leq & \Lambda-\mu_{0}\left\|\Phi_{t}\left(X_{0}\right)\right\|_{\mathcal{X}} .
\end{aligned}
$$

Hence, it follows from the variation of constants formula that, for $t \geq 0$,

$$
\left\|\Phi_{t}\left(X_{0}\right)\right\|_{\mathcal{X}} \leq \frac{\Lambda}{\mu_{0}}-e^{-\mu_{0} t}\left(\frac{\Lambda}{\mu_{0}}-\left\|\Phi_{t}\left(X_{0}\right)\right\|_{\mathcal{X}}\right),
$$

which implies that $\Phi_{t}\left(X_{0}\right) \in \Upsilon$ for any solution of (1.5) satisfying $X_{0} \in \Upsilon$ and all $t \geq 0$. Thus, the positive invariance of set $\Upsilon$ for semi-flow $\Phi$ can be verified.

Moreover, by (2.9) we can make inferences that $\limsup _{t \rightarrow \infty}\left\|\Phi_{t}\left(X_{0}\right)\right\|_{\mathcal{X}} \leq \Lambda / \mu_{0}$ for any $X_{0} \in \mathcal{X}$. Therefore, $\Phi$ is point dissipative and $\Upsilon$ attracts all points in $\mathcal{X}$. This completes the proof. 
Proposition 2.2 If $X_{0} \in \mathcal{X}$ and $\left\|X_{0}\right\|_{\mathcal{X}} \leq M$ for some constant $M \geq \Lambda / \mu_{0}$, then the following statements hold for $t \geq 0$ :

(i) $0 \leq S(t), V(t), \int_{0}^{\infty} e(t, a) \mathrm{d} a, \int_{0}^{\infty} \frac{i(t, a)}{1+\alpha i(t, a)} \mathrm{d} a, \int_{0}^{\infty} j(t, a) \mathrm{d} a \leq M$;

(ii) $e(t, 0) \leq\left(\bar{\beta}_{1}+\bar{\beta}_{2}+\bar{\beta}_{3}+\bar{\beta}_{4}\right) M^{2}, i(t, 0) \leq \bar{\gamma}_{1} M, j(t, 0) \leq\left(\bar{\gamma}_{2}+\bar{\xi}\right) M$.

Proposition 2.3 Let $C \in \mathcal{X}$ be bounded, then:

(i) $\Phi_{t}(C)$ is bounded;

(ii) $\Phi_{t}$ is eventually bounded on $C$.

\section{Asymptotic smoothness}

In order to obtain global properties of the semi-flow $\Phi(t)_{t>0}$, it is necessary to prove that the semi-flow is asymptotically smooth. Before giving the results, we first introduce some lemmas for later use.

Lemma 3.1 ([8]) Let $D \subseteq R$. For $j=1,2$, suppose $f_{j}: D \rightarrow R$ is a bounded Lipschitz continuous function with bound $K_{j}$ and Lipschitz coefficient $M_{j}$. Then the product function $f_{1} f_{2}$ is Lipschitz with coefficient $K_{1} M_{2}+K_{2} M_{1}$.

The definition of asymptotic smoothness is as follows:

Definition 3.1 ([25]) A semi-flow $\Phi\left(t, X_{0}\right):=R^{+} \times \mathcal{X} \rightarrow \mathcal{X}$ is said to be asymptotically smooth, if, for any nonempty, closed bounded set $B \subset \mathcal{X}$ for which $\Phi(t, B) \subset B$, there is a compact set $B_{0} \subset B$ such that $B_{0}$ attracts $B$.

In order to prove the asymptotic smoothness of the semi-flow, we will apply the following results, which is based on Lemma 3.2.3 in [25].

Lemma $3.2([21,22])$ If the following two conditions hold then the semi-flow $\Phi\left(t, X_{0}\right)=$ $\phi\left(t, X_{0}\right)+\varphi\left(t, X_{0}\right): R^{+} \times \mathcal{X} \rightarrow \mathcal{X}$ is asymptotically smooth in $\mathcal{X}$.

(i) There exists a continuous function $w: R^{+} \times R^{+} \rightarrow R^{+}$such that $w(t, h) \rightarrow 0$ as $t \rightarrow \infty$ and $\left\|\varphi\left(t, X_{0}\right)\right\|_{\mathcal{X}} \leq w(t, h)$ if $\left\|X_{0}\right\|_{\mathcal{X}} \leq h$;

(ii) For $t \geq 0, \phi\left(t, X_{0}\right)$ is completely continuous.

To verify that the two conditions are fulfilled for system (1.5), we decompose $\Phi: R^{+} \times$ $\mathcal{X} \rightarrow \mathcal{X}$ into the following two operators $\phi\left(t, X_{0}\right), \varphi\left(t, X_{0}\right): R^{+} \times X \rightarrow X$. Let $\phi\left(t, X_{0}\right):=$ $(S(t), V(t), \tilde{e}(t, \cdot), \tilde{i}(t, \cdot), \tilde{j}(t, \cdot))$ and $\varphi\left(t, X_{0}\right):=\left(0,0, \tilde{\varphi}_{e}(t, \cdot), \tilde{\varphi}_{i}(t, \cdot), \tilde{\varphi}_{j}(t, \cdot)\right)$ where

$$
\begin{aligned}
& \tilde{\varphi}_{e}(t, a):=\left\{\begin{array}{ll}
0 & \text { for } 0 \leq a \leq t, \\
e(t, a) & \text { for } 0 \leq t \leq a
\end{array} \text { and } \tilde{e}(t, a):= \begin{cases}e(t, a) & \text { for } 0 \leq a \leq t, \\
0 & \text { for } 0 \leq t \leq a,\end{cases} \right. \\
& \tilde{\varphi}_{i}(t, a):=\left\{\begin{array}{ll}
0 & \text { for } 0 \leq a \leq t, \\
i(t, a) & \text { for } 0 \leq t \leq a
\end{array} \text { and } \tilde{i}(t, a):= \begin{cases}i(t, a) & \text { for } 0 \leq a \leq t, \\
0 & \text { for } 0 \leq t \leq a,\end{cases} \right. \\
& \tilde{\varphi}_{j}(t, a):=\left\{\begin{array}{ll}
0 & \text { for } 0 \leq a \leq t, \\
j(t, a) & \text { for } 0 \leq t \leq a
\end{array} \text { and } \tilde{j}(t, a):= \begin{cases}j(t, a) & \text { for } 0 \leq a \leq t, \\
0 & \text { for } 0 \leq t \leq a .\end{cases} \right.
\end{aligned}
$$

Then we have $\Phi\left(t, X_{0}\right)=\varphi\left(t, X_{0}\right)+\phi\left(t, X_{0}\right)$ for all $t \geq 0$. In order to verify that condition (i) of Lemma 3.2 holds true, we turn to a proof of the following proposition. 
Proposition $3.1([26])$ For $h>0$, let $w(t, h)=h e^{-\mu_{0} t}$. Then $\lim _{t \rightarrow \infty} w(t, h)=0$ and $\left\|\varphi\left(t, X_{0}\right)\right\|_{X} \leq w(t, h)$ if $\left\|X_{0}\right\|_{X} \leq h$.

Proof Obviously, $\lim _{t \rightarrow \infty} w(t, h)=0$. For $X_{0} \in \Upsilon$ and $\left\|X_{0}\right\|_{X} \leq h$, we have

$$
\begin{aligned}
\left\|\varphi\left(t, X_{0}\right)\right\|_{X}= & |0|+|0|+\int_{0}^{\infty}\left|\tilde{\varphi}_{e}(t, a)\right| \mathrm{d} a+\int_{0}^{\infty}\left|\tilde{\varphi}_{i}(t, a)\right| \mathrm{d} a+\int_{0}^{\infty}\left|\tilde{\varphi}_{j}(t, a)\right| \mathrm{d} a \\
= & \int_{t}^{\infty}\left|\varphi_{e}(a-t) \frac{B_{1}(a)}{B_{1}(a-t)}\right| \mathrm{d} a+\int_{t}^{\infty}\left|\varphi_{i}(a-t) \frac{B_{2}(a)}{B_{2}(a-t)}\right| \mathrm{d} a \\
& +\int_{t}^{\infty}\left|\varphi_{j}(a-t) \frac{B_{3}(a)}{B_{3}(a-t)}\right| \mathrm{d} a \\
= & \int_{0}^{\infty}\left|\varphi_{e}(\tau) \frac{B_{1}(t+\tau)}{B_{1}(\tau)}\right| \mathrm{d} \tau+\int_{0}^{\infty}\left|\varphi_{i}(\tau) \frac{B_{2}(t+\tau)}{B_{2}(\tau)}\right| \mathrm{d} \tau \\
& +\int_{0}^{\infty}\left|\varphi_{j}(\tau) \frac{B_{3}(t+\tau)}{B_{3}(\tau)}\right| \mathrm{d} \tau \\
= & \int_{0}^{\infty}\left|\varphi_{e}(\tau) \exp \left(-\int_{\tau}^{t+\tau} \theta_{1}(v) \mathrm{d} v\right)\right| \mathrm{d} \tau \\
& +\int_{0}^{\infty}\left|\varphi_{i}(\tau) \exp \left(-\int_{\tau}^{t+\tau} \theta_{2}(v) \mathrm{d} v\right)\right| \mathrm{d} \tau \\
& +\int_{0}^{\infty}\left|\varphi_{j}(\tau) \exp \left(-\int_{\tau}^{t+\tau} \theta_{3}(v) \mathrm{d} v\right)\right| \mathrm{d} \tau .
\end{aligned}
$$

By (iii) of Assumption $1, \theta_{1}(a), \theta_{2}(a), \theta_{2}(a) \geq \mu_{0}$ for $a \geq 0$, we have

$$
\begin{aligned}
\left\|\varphi\left(t, X_{0}\right)\right\|_{X} & \leq e^{-\mu_{0} t}\left(|0|+|0|+\int_{0}^{\infty}\left|\varphi_{e}(\tau)\right| \mathrm{d} \tau+\int_{0}^{\infty}\left|\varphi_{i}(\tau)\right| \mathrm{d} \tau+\int_{0}^{\infty}\left|\varphi_{j}(\tau)\right| \mathrm{d} \tau\right) \\
& =e^{-\mu_{0} t}\left\|X_{0}\right\|_{X} \leq h e^{-\mu_{0} t} \equiv w(t, h) .
\end{aligned}
$$

This completes the proof.

To verify (ii) of Lemma 3.2, we need to give the following lemma.

Lemma 3.3 ([27]) Let $K \subset L^{p}(0, \infty)$ be closed and bounded where $p \geq 1$. Then $K$ is compact if the following conditions hold true.

(1) $\sup _{f \in K} \int_{0}^{\infty} f(a) \mathrm{d} a<\infty$.

(2) $\lim _{r \rightarrow \infty} \int_{r}^{\infty} f(a) \mathrm{d} a \rightarrow 0$ uniformly in $f \in K$.

(3) $\lim _{h \rightarrow 0^{+}} \int_{0}^{\infty}|f(a+h)-f(a)| \mathrm{d} a \rightarrow 0$ uniformly in $f \in K$.

(4) $\lim _{h \rightarrow 0^{+}} \int_{0}^{h} f(a) \mathrm{d} a \rightarrow 0$ uniformly in $f \in K$.

Proposition $3.2([27])$ For $t \geq 0, \phi\left(t, X_{0}\right)$ is completely continuous.

Proof From Lemma 3.3, for any closed and bounded set $B \subset X$, we see that $\phi(t, B)$ is compact. According to Proposition 2.2, $S(t)$ and $V(t)$ remain in the compact set $\left[0, \Lambda / \mu_{0}\right] \subset$ $[0, M]$, where $M \geq \Lambda / \mu_{0}$ is the bound for $B$. Thus, it is only to show that $\tilde{e}(t, a), \tilde{i}(t, a)$ and $\tilde{j}(t, a)$ remain in a precompact subset of $L_{+}^{1}(0, \infty)$, which is independent of $X_{0} \in \Upsilon$. 
Now, from (2.2) and (3.1) we have

$$
0 \leq \tilde{e}(t, a)= \begin{cases}e(t-a, 0) B_{1}(a) & \text { for } 0 \leq a<t \\ 0 & \text { for } 0 \leq t \leq a\end{cases}
$$

Then combining (i) of Proposition 2.2, we have

$$
\tilde{e}(t, a) \leq\left(\bar{\beta}_{1}+\bar{\beta}_{2}+\bar{\beta}_{3}+\bar{\beta}_{4}\right) M^{2} e^{-\mu_{0} a}
$$

which implies that (1), (2) and (4) in Lemma 3.3 are satisfied. To check condition (3), for sufficiently small $h \in(0, t)$, we have

$$
\begin{aligned}
\int_{0}^{\infty}|\tilde{e}(t, a+h)-\tilde{e}(t, a)| \mathrm{d} a= & \int_{0}^{t}|e(t, a+h)-e(t, a)| \mathrm{d} a \\
= & \int_{0}^{t-h}\left|e(t-a-h, 0) B_{1}(a+h)-e(t-a, 0) B_{1}(a)\right| \mathrm{d} a \\
& +\int_{t-h}^{t}\left|0-e(t-a, 0) B_{1}(a)\right| \mathrm{d} a \\
\leq & \int_{0}^{t-h} e(t-a-h, 0)\left|B_{1}(a+h)-B_{1}(a)\right| \mathrm{d} a \\
& +\int_{0}^{t-h} B_{1}(a)|e(t-a-h, 0)-e(t-a, 0)| \mathrm{d} a \\
& +\int_{t-h}^{t}\left|e(t-a, 0) B_{1}(a)\right| \mathrm{d} a .
\end{aligned}
$$

Recall that $0 \leq B_{1}(a) \leq e^{-\mu_{0} a} \leq 1$ and $B_{1}(a)$ is non-increasing function with respect to $a$, it follows that

$$
\begin{aligned}
\int_{0}^{t-h}\left|B_{1}(a+h)-B_{1}(a)\right| \mathrm{d} a & =\int_{0}^{t-h} B_{1}(a) \mathrm{d} a-\int_{0}^{t-h} B_{1}(a+h) \mathrm{d} a \\
& =\int_{0}^{t-h} B_{1}(a) \mathrm{d} a-\int_{h}^{t} B_{1}(a) \mathrm{d} a \\
& =\int_{0}^{t-h} B_{1}(a) \mathrm{d} a-\int_{h}^{t-h} B_{1}(a) \mathrm{d} a-\int_{t-h}^{t} B_{1}(a) \mathrm{d} a \\
& =\int_{0}^{h} B_{1}(a) \mathrm{d} a-\int_{t-h}^{t} B_{1}(a) \mathrm{d} a \leq h .
\end{aligned}
$$

Hence, from (ii) of Proposition 2.2, we have

$$
\int_{0}^{\infty}|\tilde{e}(t, a+h)-\tilde{e}(t, a)| \mathrm{d} a \leq 2\left(\bar{\beta}_{1}+\bar{\beta}_{2}+\bar{\beta}_{3}+\bar{\beta}_{4}\right) M^{2} h+\Delta
$$

where

$$
\Delta=\int_{0}^{t-h} B_{1}(a)|e(t-a-h, 0)-e(t-a, 0)| \mathrm{d} a .
$$


From (i) of Proposition 2.2, we find that $|\mathrm{d} S(t) / \mathrm{d} t|$ is bounded by $M_{S}=\Lambda+(\mu+p) M+$ $\bar{\beta}_{1} M^{2}+\bar{\beta}_{3} M^{2}$ and $|\mathrm{d} V(t) / \mathrm{d} t|$ is bounded by $M_{V}=(\mu+p) M+\bar{\beta}_{2} M^{2}+\bar{\beta}_{4} M^{2}$. Therefore, $S(\cdot)$ and $V(\cdot)$ are Lipschitz on $[0, \infty)$ with coefficients $M_{S}$ and $M_{V}$. By Lemma 3.1 of [28], there exist two Lipschitz coefficients $M_{I_{1}}, M_{I_{2}}, M_{J_{1}}, M_{J_{2}}$ for $\int_{0}^{\infty} \beta_{1}(a) i(\cdot, a) \mathrm{d} a, \int_{0}^{\infty} \beta_{2}(a) i(\cdot, a) \mathrm{d} a$, $\int_{0}^{\infty} \beta_{3}(a) j(\cdot, a) \mathrm{d} a, \int_{0}^{\infty} \beta_{4}(a) j(\cdot, a) \mathrm{d} a$, respectively. Thus, $\int_{0}^{\infty} \beta_{1}(a) i(\cdot, a) S(\cdot) \mathrm{d} a, \int_{0}^{\infty} \beta_{2}(a) i(\cdot$, a) $V(\cdot) \mathrm{d} a, \int_{0}^{\infty} \beta_{3}(a) j(\cdot, a) S(\cdot) \mathrm{d} a, \int_{0}^{\infty} \beta_{4}(a) j(\cdot, a) V(\cdot) \mathrm{d} a$ are Lipschitz continuous on $[0, \infty)$ with coefficients $M_{S I}=K M_{I_{1}}+K M_{S}, M_{S J}=K M_{J_{1}}+K M_{S}, M_{V I}=K M_{I_{2}}+K M_{V}, M_{V J}=K M_{J_{2}}+$ $K M_{V}$, respectively. Denote $M=M_{S I}+M_{S I}+M_{V I}+M_{V I}$. Then

$$
\Delta \leq M h \int_{0}^{t-h} e^{-\mu_{0} a} \mathrm{~d} a \leq \frac{M h}{\mu_{0}} .
$$

Finally, by (3.4) and (3.5), we have

$$
\int_{0}^{\infty}|\tilde{e}(t, a+h)-\tilde{e}(t, a)| \mathrm{d} a \leq\left(2 \bar{\beta} M^{2}+\frac{M}{\mu_{0}}\right) h,
$$

where $\bar{\beta}=\bar{\beta}_{1}+\bar{\beta}_{2}+\bar{\beta}_{3}+\bar{\beta}_{4}$. The right hand of (3.6) converges uniformly to 0 as $h \rightarrow 0$ and condition (3) is proved for $\tilde{e}(t, a)$. Noting that (3.4) holds for any $X_{0} \in B$, thus, $\tilde{e}(t, a)$ remains in a precompact subset $B_{\tilde{e}}$ of $L_{+}^{1}(0, \infty)$. Similarly, $\tilde{i}(t, a)$ and $\tilde{j}(t, a)$ remain in a precompact subset $B_{\tilde{i}}, B_{\tilde{j}}$ of $L_{+}^{1}(0, \infty)$, respectively. Thus, the proof is completed.

From Propositions 3.1 and 3.2, we apply Lemma 3.2 and conclude that the following theorem holds.

Theorem 3.1 The semi-flow $\Phi(t)_{t \geq 0}$ generated by system (1.5) is asymptotically smooth.

\section{The existence of steady states}

System (1.5) always has the steady state $E_{0}=\left(S_{0}, V_{0}, 0,0,0\right)$, where

$$
S_{0}=\frac{\Lambda(\mu+\rho+\eta)}{(\mu+p)(\mu+\rho+\eta)-p \eta}, \quad V_{0}=\frac{p \Lambda}{(\mu+p)(\mu+\rho+\eta)-p \eta} .
$$

Define the basic reproduction number as follows

$$
\begin{aligned}
R_{0}:= & S_{0}\left[\int_{0}^{\infty} \gamma_{1}(a) B_{1}(a) \mathrm{d} a \int_{0}^{\infty} \beta_{1}(a) B_{2}(a) \mathrm{d} a\right. \\
& +\int_{0}^{\infty} \beta_{3}(a) B_{3}(a) \mathrm{d} a\left(\int_{0}^{\infty} \gamma_{2}(a) B_{1}(a) \mathrm{d} a\right. \\
& \left.\left.+\int_{0}^{\infty} \gamma_{1}(a) B_{1}(a) \mathrm{d} a \int_{0}^{\infty} \xi(a) B_{2}(a) \mathrm{d} a\right)\right] \\
& +V_{0}\left[\int_{0}^{\infty} \gamma_{1}(a) B_{1}(a) \mathrm{d} a \int_{0}^{\infty} \beta_{2}(a) B_{2}(a) \mathrm{d} a\right. \\
& +\int_{0}^{\infty} \beta_{4}(a) B_{3}(a) \mathrm{d} a\left(\int_{0}^{\infty} \gamma_{2}(a) B_{1}(a) \mathrm{d} a\right. \\
& \left.\left.+\int_{0}^{\infty} \gamma_{1}(a) B_{1}(a) \mathrm{d} a \int_{0}^{\infty} \xi(a) B_{2}(a) \mathrm{d} a\right)\right] .
\end{aligned}
$$


Now we consider the existence of positive steady state of system (1.5). The steady state $\left(S^{*}, V^{*}, e^{*}(\cdot), i^{*}(\cdot), j^{*}(\cdot)\right)$ of system (1.5) satisfies the following equalities:

$$
\begin{aligned}
& \Lambda-(\mu+p) S^{*}+\eta V^{*}-S^{*} \int_{0}^{\infty}\left(\frac{\beta_{1}(a) i^{*}(a)}{1+\alpha i^{*}(a)}+\beta_{3}(a) j^{*}(a)\right) \mathrm{d} a=0, \\
& p S^{*}-(\mu+\rho+\eta) V^{*}-V^{*} \int_{0}^{\infty}\left(\frac{\beta_{2}(a) i^{*}(a)}{1+\alpha i^{*}(a)}+\beta_{4}(a) j^{*}(a)\right) \mathrm{d} a=0, \\
& \frac{d e^{*}(a)}{\mathrm{d} a}=-\theta_{1}(a) e^{*}(a), \\
& \frac{d i^{*}(a)}{\mathrm{d} a}=-\theta_{2}(a) i^{*}(a), \\
& \frac{d j^{*}(a)}{\mathrm{d} a}=-\theta_{3}(a) j^{*}(a), \\
& e^{*}(0)=S^{*} \int_{0}^{\infty}\left(\frac{\beta_{1}(a) i^{*}(a)}{1+\alpha i^{*}(a)}+\beta_{3}(a) j^{*}(a)\right) \mathrm{d} a \\
& \quad+V^{*} \int_{0}^{\infty}\left(\frac{\beta_{2}(a) i^{*}(a)}{1+\alpha i^{*}(a)}+\beta_{4}(a) j^{*}(a)\right) \mathrm{d} a, \\
& i^{*}(0)=\int_{0}^{\infty} \gamma_{1}(a) e^{*}(a) \mathrm{d} a, \\
& j^{*}(0)=\int_{0}^{\infty} \gamma_{2}(a) e^{*}(a) \mathrm{d} a+\int_{0}^{\infty} \xi(a) i^{*}(a) \mathrm{d} a .
\end{aligned}
$$

Solving the third, fourth and fifth equations of (4.1) yields

$$
e^{*}(a)=e^{*}(0) B_{1}(a), \quad i^{*}(a)=i^{*}(0) B_{2}(a), \quad j^{*}(a)=j^{*}(0) B_{3}(a) .
$$

From the first and second equations of (4.1), it is easy to get

$$
\begin{aligned}
\Lambda & -\mu S^{*}-(\mu+\rho) V^{*}-S^{*}\left(\Lambda-\mu S^{*}-(\mu+\rho) V^{*}\right) f_{1}\left(S^{*}, V^{*}\right) \\
& -V^{*}\left(\Lambda-\mu S^{*}-(\mu+\rho) V^{*}\right) f_{2}\left(S^{*}, V^{*}\right)=0,
\end{aligned}
$$

where

$$
\begin{aligned}
& f_{1}\left(S^{*}, V^{*}\right)=\int_{0}^{\infty}\left(\frac{K_{1} K_{3}(a)}{1+\alpha K_{1}\left(\Lambda-\mu S^{*}-(\mu+\rho) V^{*}\right) B_{2}(a)}+\left(K_{2}+K_{1} K_{7}\right) K_{4}(a)\right) \mathrm{d} a \\
& f_{2}\left(S^{*}, V^{*}\right)=\int_{0}^{\infty}\left(\frac{K_{1} K_{5}(a)}{1+\alpha K_{1}\left(\Lambda-\mu S^{*}-(\mu+\rho) V^{*}\right) B_{2}(a)}+\left(K_{2}+K_{1} K_{7}\right) K_{6}(a)\right) \mathrm{d} a
\end{aligned}
$$

and

$$
\begin{aligned}
& K_{1}=\int_{0}^{\infty} \gamma_{1}(a) B_{1}(a) \mathrm{d} a, \quad K_{2}=\int_{0}^{\infty} \gamma_{2}(a) B_{1}(a) \mathrm{d} a \\
& K_{3}(a)=\beta_{1}(a) B_{2}(a), \quad K_{4}(a)=\beta_{3}(a) B_{3}(a), \quad K_{5}(a)=\beta_{2}(a) B_{2}(a), \\
& K_{6}(a)=\beta_{4}(a) B_{3}(a), \quad K_{7}=\int_{0}^{\infty} \xi(a) B_{2}(a) \mathrm{d} a .
\end{aligned}
$$


Let

$$
\begin{aligned}
F\left(S^{*}, V^{*}\right)= & \Lambda-\mu S^{*}-(\mu+\rho) V^{*}-S^{*}\left(\Lambda-\mu S^{*}-(\mu+\rho) V^{*}\right) f_{1}\left(S^{*}, V^{*}\right) \\
& -V^{*}\left(\Lambda-\mu S^{*}-(\mu+\rho) V^{*}\right) f_{2}\left(S^{*}, V^{*}\right) .
\end{aligned}
$$

Let $V^{*}=V_{0}$. It is easy to see that $F\left(0, V_{0}\right)=\Lambda-(\mu+\rho) V_{0}-V_{0}\left(\Lambda-(\mu+\rho) V_{0}\right) f_{2}\left(0, V_{0}\right)$ and $F\left(S_{0}, V_{0}\right)=0$. When $0 \leq S^{*} \leq S_{0}, \Lambda-\mu S^{*}-(\mu+\rho) V_{0} \neq 0$, then we get

$$
S^{*} f_{1}\left(S^{*}, V_{0}\right)+V_{0} f_{2}\left(S^{*}, V_{0}\right)-1=0 .
$$

Let $g\left(S^{*}\right)=S^{*} f_{1}\left(S^{*}, V_{0}\right)+V_{0} f_{2}\left(S^{*}, V_{0}\right)-1$, where $g(0)=V_{0} f_{2}\left(0, V_{0}\right)-1<V_{0} f_{2}\left(S^{*}, V_{0}\right)-1<0$ and $g\left(S_{0}\right)=S_{0} f_{1}\left(S_{0}, V_{0}\right)+V_{0} f_{2}\left(S_{0}, V_{0}\right)-1$. Note that

$$
g^{\prime}\left(S^{*}\right)=f_{1}\left(S^{*}, V_{0}\right)+S^{*} \frac{\mathrm{d} f_{1}\left(S^{*}, V_{0}\right)}{\mathrm{d} S^{*}}+V_{0} \frac{\mathrm{d} f_{2}\left(S^{*}, V_{0}\right)}{\mathrm{d} S^{*}}>0,
$$

for

$$
\begin{aligned}
& \frac{\mathrm{d} f_{1}\left(S^{*}, V_{0}\right)}{\mathrm{d} S^{*}}=\int_{0}^{\infty} \frac{\mu \alpha K_{1}^{2} K_{3}(a) B_{2}(a)}{\left(1+\alpha K_{1}\left(\Lambda-\mu S^{*}-(\mu+\rho) V_{0}\right) B_{2}(a)\right)^{2}} \mathrm{~d} a>0, \\
& \frac{\mathrm{d} f_{2}\left(S^{*}, V_{0}\right)}{\mathrm{d} S^{*}}=\int_{0}^{\infty} \frac{\mu \alpha K_{1}^{2} K_{5}(a) B_{2}(a)}{\left(1+\alpha K_{1}\left(\Lambda-\mu S^{*}-(\mu+\rho) V_{0}\right) B_{2}(a)\right)^{2}} \mathrm{~d} a>0 .
\end{aligned}
$$

It is easy to show that if $g\left(S_{0}\right)>0, g\left(S^{*}\right)=0$ has a unique positive root. Define the basic reproduction number as

$$
R_{0}=g\left(S_{0}\right)+1=S_{0} f_{1}\left(S_{0}, V_{0}\right)+V_{0} f_{2}\left(S_{0}, V_{0}\right),
$$

which means the number of newly infected individuals produced by one infected individual during its period of disease. Therefore, if $R_{0}>1$, there exists a unique positive steady state $E^{*}$ of system (1.5), where $E^{*}=\left(S^{*}, V^{*}, e^{*}(a), i^{*}(a), j^{*}(a)\right)$.

From the above discussions, we have the following theorem.

Theorem 4.1 System (1.5) always has a steady state $E_{0}\left(S_{0}, V_{0}, 0,0,0\right)$, where $S_{0}=\Lambda(\mu+$ $\rho+\eta) /[(\mu+p)(\mu+\rho+\eta)-p \eta], V_{0}=p \Lambda /[(\mu+p)(\mu+\rho+\eta)-p \eta]$; system (1.5) has a unique positive steady state $E^{*}\left(S^{*}, V^{*}, e^{*}(\cdot), i^{*}(\cdot), j^{*}(\cdot)\right)$ if and only if $R_{0}>1$.

\section{Local stability}

This section is mainly used to prove the local stability of steady states, and to verify that the basic reproduction number is related to the stability of the steady states.

Theorem 5.1 The steady state $E_{0}$ is locally asymptotically stable if $R_{0}<1$.

Proof First, we introduce the change of variables as follows:

$$
\begin{aligned}
& s_{1}(t)=S(t)-S_{0}, \quad v_{1}(t)=V(t)-V_{0}, \quad e_{1}(t, a)=e(t, a), \\
& i_{1}(t, a)=i(t, a), \quad j_{1}(t, a)=j(t, a) .
\end{aligned}
$$


Linearizing system (1.5) at the steady state $E_{0}$ yields the following system:

$$
\begin{aligned}
& \dot{s}_{1}(t)=-(\mu+p) s_{1}(t)+\eta v_{1}(t)-S_{0} \int_{0}^{\infty}\left(\beta_{1}(a) i_{1}(t, a)+\beta_{3}(a) j_{1}(t, a)\right) \mathrm{d} a, \\
& \dot{v}_{1}(t)=p s_{1}(t)-(\mu+\rho+\eta) \nu_{1}(t)-V_{0} \int_{0}^{\infty}\left(\beta_{2}(a) i_{1}(t, a)+\beta_{4}(a) j_{1}(t, a)\right) \mathrm{d} a, \\
& \frac{\partial e_{1}(t, a)}{\partial a}+\frac{\partial e_{1}(t, a)}{\partial t}=-\theta_{1}(a) e_{1}(t, a), \\
& \frac{\partial i_{1}(t, a)}{\partial a}+\frac{\partial i_{1}(t, a)}{\partial t}=-\theta_{2}(a) i_{1}(t, a), \\
& \frac{\partial j_{1}(t, a)}{\partial a}+\frac{\partial j_{1}(t, a)}{\partial t}=-\theta_{3}(a) j_{1}(t, a), \\
& e_{1}(t, 0)=S_{0}^{\infty}\left(\beta_{1}(a) i_{1}(t, a)+\beta_{3}(a) j_{1}(t, a)\right) \mathrm{d} a \\
& \quad+V_{0}^{\infty}\left(\beta_{0}(a) i_{1}(t, a)+\beta_{4}(a) j_{1}(t, a)\right) \mathrm{d} a, \\
& i_{1}(t, 0)=\int_{0}^{\infty} \gamma_{1}(a) e_{1}(t, a) \mathrm{d} a, \\
& j_{1}(t, 0)=\int_{0}^{\infty} \gamma_{2}(a) e_{1}(t, a) \mathrm{d} a+\int_{0}^{\infty} \xi(a) i_{1}(t, a) \mathrm{d} a .
\end{aligned}
$$

Set

$$
\begin{aligned}
& s_{1}(t)=s_{1}^{0} e^{\lambda t}, \quad v_{1}(t)=v_{1}^{0} e^{\lambda t}, \quad e_{1}(t, a)=e_{1}^{0}(a) e^{\lambda t}, \\
& i_{1}(t, a)=i_{1}^{0}(a) e^{\lambda t}, \quad j_{1}(t, a)=j_{1}^{0}(a) e^{\lambda t},
\end{aligned}
$$

where $s_{1}^{0}, v_{1}^{0}, e_{1}^{0}(a), i_{1}^{0}(a), j_{1}^{0}(a)$ will be determined later. We can get

$$
\begin{aligned}
& \lambda s_{1}^{0}=-(\mu+p) s_{1}^{0}+\eta v_{1}^{0}-S_{0} \int_{0}^{\infty}\left(\beta_{1}(a) i_{1}^{0}(a)+\beta_{3}(a) j_{1}^{0}(a)\right) \mathrm{d} a \\
& \lambda v_{1}^{0}=p s_{1}^{0}-(\mu+\rho+\eta) v_{1}^{0}-V_{0} \int_{0}^{\infty}\left(\beta_{2}(a) i_{1}^{0}(a)+\beta_{4}(a) j_{1}^{0}(a)\right) \mathrm{d} a \\
& \frac{\mathrm{d} e_{1}^{0}(a)}{\mathrm{d} a}=-\left(\lambda+\theta_{1}(a)\right) e_{1}^{0}(a), \\
& \frac{\mathrm{d} i_{1}^{0}(a)}{\mathrm{d} a}=-\left(\lambda+\theta_{2}(a)\right) i_{1}^{0}(a), \\
& \frac{\mathrm{d} j_{1}^{0}(a)}{\mathrm{d} a}=-\left(\lambda+\theta_{3}(a)\right) j_{1}^{0}(a), \\
& e_{1}^{0}(0)=S_{0} \int_{0}^{\infty}\left(\beta_{1}(a) i_{1}^{0}(a)+\beta_{3}(a) j_{1}^{0}(a)\right) \mathrm{d} a \\
& \quad+V_{0} \int_{0}^{\infty}\left(\beta_{2}(a) i_{1}^{0}(a)+\beta_{4}(a) j_{1}^{0}(a)\right) \mathrm{d} a \\
& i_{1}^{0}(0)=\int_{0}^{\infty} \gamma_{1}(a) e_{1}^{0}(a) \mathrm{d} a, \\
& j_{1}^{0}(0)=\int_{0}^{\infty} \gamma_{2}(a) e_{1}^{0}(a) \mathrm{d} a+\int_{0}^{\infty} \xi(a) i_{1}^{0}(a) \mathrm{d} a .
\end{aligned}
$$


Integrating the third, fourth and fifth equations of (5.1) from 0 to $a$ yields

$$
\begin{aligned}
& e_{1}^{0}(a)=e_{1}^{0}(0) \exp \left(-\int_{0}^{a}\left(\lambda+\theta_{1}(\tau)\right) \mathrm{d} \tau\right), \\
& i_{1}^{0}(a)=i_{1}^{0}(0) \exp \left(-\int_{0}^{a}\left(\lambda+\theta_{2}(\tau)\right) \mathrm{d} \tau\right), \\
& j_{1}^{0}(a)=j_{1}^{0}(0) \exp \left(-\int_{0}^{a}\left(\lambda+\theta_{3}(\tau)\right) \mathrm{d} \tau\right) .
\end{aligned}
$$

If $e_{1}^{0}(0)=0$, then $i_{1}^{0}(0)=0, j_{1}^{0}(0)=0$. Plugging it into (5.1), we have

$$
(\lambda+\mu+p) s_{1}^{0}-\eta \nu_{1}^{0}=0, \quad(\lambda+\mu+\rho+\eta) \nu_{1}^{0}-p s_{1}^{0}=0 .
$$

For $s_{1}^{0} \neq 0$ and $v_{1}^{0} \neq 0$, it is easy to get

$$
\lambda^{2}+(2 \mu+p+\rho+\eta) \lambda+(\mu+p)(\mu+\rho+\eta)-p \eta=0 .
$$

Then

$$
\lambda=\frac{-b_{1} \pm \sqrt{b_{1}^{2}-4 b_{2}}}{2},
$$

where $b_{1}=2 \mu+p+\rho+\eta, b_{2}=(\mu+p)(\mu+\rho+\eta)-p \eta$.

If $e_{1}^{0}(0) \neq 0$,

$$
(\lambda+\mu) s_{1}^{0}+(\lambda+\mu+\rho) v_{1}^{0}+e_{1}^{0}(0)=0,
$$

where

$$
\begin{aligned}
& s_{1}^{0}=\frac{S_{0}(\lambda+\mu+\rho+\eta) f_{3}(\lambda)+\eta V_{0} f_{4}(\lambda)}{p \eta-(\lambda+\mu+p)(\lambda+\mu+\rho+\eta)} e_{1}^{0}(0), \\
& v_{1}^{0}=\frac{p S_{0} f_{3}(\lambda)+V_{0}(\lambda+\mu+p) f_{4}(\lambda)}{p \eta-(\lambda+\mu+p)(\lambda+\mu+\rho+\eta)} e_{1}^{0}(0),
\end{aligned}
$$

where

$$
\begin{aligned}
& f_{3}(\lambda)=\int_{0}^{\infty}\left(\beta_{1}(a) B_{2}(\lambda, a) U_{1}(\lambda)+\beta_{3}(a) B_{3}(\lambda, a)\left(U_{2}(\lambda)+U_{1}(\lambda) U_{7}(\lambda)\right)\right) \mathrm{d} a, \\
& f_{4}(\lambda)=\int_{0}^{\infty}\left(\beta_{2}(a) B_{2}(\lambda, a) U_{1}(\lambda)+\beta_{4}(a) B_{3}(\lambda, a)\left(U_{2}(\lambda)+U_{1}(\lambda) U_{7}(\lambda)\right)\right) \mathrm{d} a
\end{aligned}
$$

where

$$
\begin{aligned}
& U_{1}(\lambda)=\int_{0}^{\infty} \gamma_{1}(a) B_{1}(\lambda, a) \mathrm{d} a, \quad U_{2}(\lambda)=\int_{0}^{\infty} \gamma_{2}(a) B_{1}(\lambda, a) \mathrm{d} a \\
& U_{7}(\lambda)=\int_{0}^{\infty} \xi(a) B_{2}(\lambda, a) \mathrm{d} a, \quad B_{1}(\lambda, a)=\exp \left(-\int_{0}^{a}\left(\lambda+\theta_{1}(\tau)\right) \mathrm{d} \tau\right), \\
& B_{2}(\lambda, a)=\exp \left(-\int_{0}^{a}\left(\lambda+\theta_{2}(\tau)\right) \mathrm{d} \tau\right), \quad B_{3}(\lambda, a)=\exp \left(-\int_{0}^{a}\left(\lambda+\theta_{3}(\tau)\right) \mathrm{d} \tau\right) .
\end{aligned}
$$


That is,

$$
S_{0} f_{3}(\lambda)+V_{0} f_{4}(\lambda)=1
$$

Assume that $\operatorname{Re} \lambda \geq 0$, then $\left|f_{3}(\lambda)\right| \leq f_{1}\left(S_{0}, V_{0}\right)$ and $\left|f_{4}(\lambda)\right| \leq f_{2}\left(S_{0}, V_{0}\right)$ hold. Hence, the modulus of the left-hand side of Eq. (5.3) satisfies

$$
\begin{aligned}
& \left|S_{0} f_{3}(\lambda)+V_{0} f_{4}(\lambda)\right| \\
& \quad \leq\left|S_{0} f_{3}(\lambda)\right|+\left|V_{0} f_{4}(\lambda)\right|<S_{0} f_{1}\left(S_{0}, V_{0}\right)+V_{0} f_{2}\left(S_{0}, V_{0}\right)=R_{0} .
\end{aligned}
$$

It follows from (5.3) that there is a contradiction. Thus, all the roots of Eq. (5.3) have a negative real part if and only if $R_{0}<1$. Therefore, the steady state $E_{0}$ is locally asymptotically stable if $R_{0}<1$. This completes the proof.

Theorem 5.2 The steady state $E^{*}$ is locally asymptotically stable if $R_{0}>1$.

Proof Linearizing system (1.5) at the steady state $E^{*}$ under introducing the perturbation variables

$$
\begin{aligned}
& s_{2}(t)=S(t)-S^{*}, \quad v_{2}(t)=V(t)-V^{*}, \quad e_{2}(t, a)=e(t, a)-e^{*}(a), \\
& i_{2}(t, a)=i(t, a)-i^{*}(a), \quad j_{2}(t, a)=j(t, a)-j^{*}(a),
\end{aligned}
$$

we obtain the following system:

$$
\begin{aligned}
\dot{s}_{2}(t)= & -(\mu+p) s_{2}(t)+\eta v_{2}(t)-s_{2}(t) \int_{0}^{\infty} \frac{\beta_{1}(a) i^{*}(a)}{1+\alpha i^{*}(a)} \mathrm{d} a-S^{*} \int_{0}^{\infty} \frac{\beta_{1}(a) i_{2}(t, a)}{\left(1+\alpha i^{*}(a)\right)^{2}} \mathrm{~d} a \\
& -s_{2}(t) \int_{0}^{\infty} \beta_{3}(a) j^{*}(a) \mathrm{d} a-S^{*} \int_{0}^{\infty} \beta_{3}(a) j_{2}(t, a) \mathrm{d} a, \\
\dot{v}_{2}(t)= & p s_{2}(t)-(\mu+\rho+\eta) v_{2}(t)-v_{2}(t) \int_{0}^{\infty} \frac{\beta_{2}(a) i^{*}(a)}{1+\alpha i^{*}(a)} \mathrm{d} a-V^{*} \int_{0}^{\infty} \frac{\beta_{2}(a) i_{2}(t, a)}{\left(1+\alpha i^{*}(a)\right)^{2}} \mathrm{~d} a \\
& -v_{2}(t) \int_{0}^{\infty} \beta_{4}(a) j^{*}(a) \mathrm{d} a-V^{*} \int_{0}^{\infty} \beta_{4}(a) j_{2}(t, a) \mathrm{d} a, \\
\frac{\partial e_{2}(t, a)}{\partial a}+ & \frac{\partial e_{2}(t, a)}{\partial t}=-\theta_{1}(a) e_{2}(t, a), \\
\frac{\partial i_{2}(t, a)}{\partial a}+ & \frac{\partial i_{2}(t, a)}{\partial t}=-\theta_{2}(a) i_{2}(t, a), \\
\frac{\partial j_{2}(t, a)}{\partial a}+ & \frac{\partial j_{1}(t, a)}{\partial t}=-\theta_{3}(a) j_{2}(t, a), \\
e_{2}(t, 0)= & s_{2}(t) \int_{0}^{\infty} \frac{\beta_{1}(a) i^{*}(a)}{1+\alpha i^{*}(a)} \mathrm{d} a+S^{*} \int_{0}^{\infty} \frac{\beta_{1}(a) i_{2}(t, a)}{\left(1+\alpha i^{*}(a)\right)^{2}} \mathrm{~d} a+s_{2}(t) \int_{0}^{\infty} \beta_{3}(a) j^{*}(a) \mathrm{d} a \\
& +S^{*} \int_{0}^{\infty} \beta_{3}(a) j_{2}(t, a) \mathrm{d} a+v_{2}(t) \int_{0}^{\infty} \frac{\beta_{2}(a) i^{*}(a)}{1+\alpha i^{*}(a)} \mathrm{d} a+V^{*} \int_{0}^{\infty} \frac{\beta_{2}(a) i_{2}(t, a)}{\left(1+\alpha i^{*}(a)\right)^{2}} \mathrm{~d} a \\
& +v_{2}(t) \int_{0}^{\infty} \beta_{4}(a) j^{*}(a) \mathrm{d} a+V^{*} \int_{0}^{\infty} \beta_{4}(a) j_{2}(t, a) \mathrm{d} a, \\
i_{2}(t, 0)= & \int_{0}^{\infty} \gamma_{1}(a) e_{2}(t, a) \mathrm{d} a,
\end{aligned}
$$




$$
j_{2}(t, 0)=\int_{0}^{\infty} \gamma_{2}(a) e_{2}(t, a) \mathrm{d} a+\int_{0}^{\infty} \xi(a) i_{2}(t, a) \mathrm{d} a .
$$

Set

$$
\begin{aligned}
& s_{2}(t)=s_{2}^{0} e^{\lambda t}, \quad \nu_{2}(t)=v_{2}^{0} e^{\lambda t}, \quad e_{2}(t, a)=e_{2}^{0}(a) e^{\lambda t}, \\
& i_{2}(t, a)=i_{2}^{0}(a) e^{\lambda t}, \quad j_{2}(t, a)=j_{2}^{0}(a) e^{\lambda t},
\end{aligned}
$$

where $s_{2}^{0}, v_{2}^{0}, e_{2}^{0}(a), i_{2}^{0}(a), j_{2}^{0}(a)$ will be determined later. We get

$$
\begin{aligned}
& \lambda s_{2}^{0}=-(\mu+p) s_{2}^{0}+\eta v_{2}^{0}-S^{*} \int_{0}^{\infty} \frac{\beta_{1}(a) i_{2}^{0}(a)}{\left(1+\alpha i^{*}(a)\right)^{2}} \mathrm{~d} a-s_{2}^{0} \int_{0}^{\infty} \frac{\beta_{1}(a) i^{*}(a)}{1+\alpha i^{*}(a)} \mathrm{d} a \\
&-S^{*} \int_{0}^{\infty} \beta_{3}(a) j_{2}^{0}(a) \mathrm{d} a-s_{2}^{0} \int_{0}^{\infty} \beta_{3}(a) j^{*}(a) \mathrm{d} a, \\
& \lambda v_{2}^{0}= p s_{2}^{0}-(\mu+\rho+\eta) v_{2}^{0}-V^{*} \int_{0}^{\infty} \frac{\beta_{2}(a) i_{2}^{0}(a)}{\left(1+\alpha i^{*}(a)\right)^{2}} \mathrm{~d} a-v_{2}^{0} \int_{0}^{\infty} \frac{\beta_{2}(a) i^{*}(a)}{1+\alpha i^{*}(a)} \mathrm{d} a \\
&-V^{*} \int_{0}^{\infty} \beta_{4}(a) j_{2}^{0}(a) \mathrm{d} a-v_{2}^{0} \int_{0}^{\infty} \beta_{4}(a) j^{*}(a) \mathrm{d} a, \\
& \frac{\mathrm{d} e_{2}^{0}(a)}{\mathrm{d} a}=-\left(\lambda+\theta_{1}(a)\right) e_{2}^{0}(a), \\
& \frac{\mathrm{d} i_{2}^{0}(a)}{\mathrm{d} a}=-\left(\lambda+\theta_{2}(a)\right) i_{2}^{0}(a), \\
& \frac{\mathrm{d} j_{2}^{0}(a)}{\mathrm{d} a}=-\left(\lambda+\theta_{3}(a)\right) j_{2}^{0}(a), \\
& e_{2}^{0}(0)= s_{2}^{0} \int_{0}^{\infty} \frac{\beta_{1}(a) i^{*}(a)}{1+\alpha i^{*}(a)} \mathrm{d} a+S^{*} \int_{0}^{\infty} \frac{\beta_{1}(a) i_{2}^{0}(a)}{\left(1+\alpha i^{*}(a)\right)^{2}} \mathrm{~d} a \\
&+s_{2}^{0} \int_{0}^{\infty} \beta_{3}(a) j^{*}(a) \mathrm{d} a+S^{*} \int_{0}^{\infty} \beta_{3}(a) j_{2}^{0}(a) \mathrm{d} a \\
&+v_{2}^{0} \int_{0}^{\infty} \frac{\beta_{2}(a) i^{*}(a)}{1+\alpha i^{*}(a)} \mathrm{d} a+V^{*} \int_{0}^{\infty} \frac{\beta_{2}(a) i_{2}^{0}(a)}{\left(1+\alpha i^{*}(a)\right)^{2}} \mathrm{~d} a \\
&+v_{2}^{0} \int_{0}^{\infty} \beta_{4}(a) j^{*}(a) \mathrm{d} a+V^{*} \int_{0}^{\infty} \beta_{4}(a) j_{2}^{0}(a) \mathrm{d} a, \\
& i_{2}^{0}(0)= \int_{0}^{\infty} \gamma_{1}(a) e_{2}^{0}(a) \mathrm{d} a, \\
& j_{2}^{0}(0)= \int_{0}^{\infty} \gamma_{2}(a) e_{2}^{0}(a) \mathrm{d} a+\int_{0}^{\infty} \xi(a) i_{2}^{0}(a) \mathrm{d} a . \\
&
\end{aligned}
$$

Integrating the third, fourth and fifth equations of (5.4) from 0 to $a$ yields

$$
\begin{aligned}
& e_{2}^{0}(a)=e_{2}^{0}(0) \exp \left(-\int_{0}^{a}\left(\lambda+\theta_{1}(\tau)\right) \mathrm{d} \tau\right), \\
& i_{2}^{0}(a)=i_{2}^{0}(0) \exp \left(-\int_{0}^{a}\left(\lambda+\theta_{2}(\tau)\right) \mathrm{d} \tau\right), \\
& j_{2}^{0}(a)=j_{2}^{0}(0) \exp \left(-\int_{0}^{a}\left(\lambda+\theta_{3}(\tau)\right) \mathrm{d} \tau\right) .
\end{aligned}
$$


The characteristic equation is

$$
(\lambda+\mu) s_{2}^{0}+(\lambda+\mu+\rho) v_{2}^{0}+e_{2}^{0}(0)=0
$$

where

$$
\begin{aligned}
& s_{2}^{0}=\frac{S^{*}\left(\lambda+\mu+\rho+\eta+f_{6}\left(e^{*}\right)\right) f_{3}\left(e^{*}, \lambda\right)+\eta V^{*} f_{4}\left(e^{*}, \lambda\right)}{p \eta-\left(\lambda+\mu+p+f_{5}\left(e^{*}\right)\right)\left(\lambda+\mu+\rho+\eta+f_{6}\left(e^{*}\right)\right)} e_{2}^{0}, \\
& v_{2}^{0}=\frac{p S^{*} f_{3}\left(e^{*}, \lambda\right)+V^{*}\left(\lambda+\mu+p+f_{5}\left(e^{*}\right)\right) f_{4}\left(e^{*}, \lambda\right)}{p \eta-\left(\lambda+\mu+p+f_{5}\left(e^{*}\right)\right)\left(\lambda+\mu+\rho+\eta+f_{6}\left(e^{*}\right)\right)} e_{2}^{0},
\end{aligned}
$$

where

$$
\begin{aligned}
& f_{3}\left(e^{*}, \lambda\right)=\int_{0}^{\infty}\left(\frac{\beta_{1}(a) B_{2}(\lambda, a) U_{1}(\lambda)}{\left(1+\alpha K_{1} e^{*}(0) B_{2}(a)\right)^{2}}+\beta_{3}(a) B_{3}(\lambda, a)\left(U_{2}(\lambda)+U_{1}(\lambda) U_{7}(\lambda)\right)\right) \mathrm{d} a \\
& f_{4}\left(e^{*}, \lambda\right)=\int_{0}^{\infty}\left(\frac{\beta_{2}(a) B_{2}(\lambda, a) U_{1}(\lambda)}{\left(1+\alpha K_{1} e^{*}(0) B_{2}(a)\right)^{2}}+\beta_{4}(a) B_{3}(\lambda, a)\left(U_{2}(\lambda)+U_{1}(\lambda) U_{7}(\lambda)\right)\right) \mathrm{d} a \\
& f_{5}\left(e^{*}\right)=\int_{0}^{\infty}\left(\frac{K_{1} \beta_{1}(a) B_{2}(a)}{1+\alpha K_{1} e^{*}(0) B_{2}(a)}+\beta_{3}(a) B_{3}(a)\left(K_{2}+K_{1} K_{7}\right)\right) \mathrm{d} a \\
& f_{6}\left(e^{*}\right)=\int_{0}^{\infty}\left(\frac{K_{1} \beta_{2}(a) B_{2}(a)}{1+\alpha K_{1} e^{*}(0) B_{2}(a)}+\beta_{4}(a) B_{3}(a)\left(K_{2}+K_{1} K_{7}\right)\right) \mathrm{d} a
\end{aligned}
$$

That is,

$$
\begin{gathered}
\frac{(\lambda+\mu)\left(\lambda+\mu+\rho+\eta+f_{6}\left(e^{*}\right)\right)+p(\lambda+\mu+\rho)}{\left(\lambda+\mu+f_{5}\left(e^{*}\right)\right)\left(\lambda+\mu+\rho+\eta+f_{6}\left(e^{*}\right)\right)+p\left(\lambda+\mu+\rho+f_{6}\left(e^{*}\right)\right)} S^{*} f_{3}\left(e^{*}, \lambda\right) \\
+\frac{\left(\lambda+\mu+p+f_{5}\left(e^{*}\right)\right)(\lambda+\mu+\rho)+\eta(\lambda+\mu)}{\left(\lambda+\mu+p+f_{5}\left(e^{*}\right)\right)\left(\lambda+\mu+\rho+f_{6}\left(e^{*}\right)\right)+\eta\left(\lambda+\mu+f_{5}\left(e^{*}\right)\right)} V^{*} f_{4}\left(e^{*}, \lambda\right)
\end{gathered}
$$

$=1$.

Assume that $\operatorname{Re} \lambda \geq 0$, then $\left|f_{3}\left(e^{*}, \lambda\right)\right| \leq f_{5}\left(e^{*}\right)$ and $\left|f_{4}\left(e^{*}, \lambda\right)\right| \leq f_{6}\left(e^{*}\right)$ hold. Hence, the modulus of the left-hand side of Eq. (5.6) satisfies

$$
\begin{aligned}
& \mid \frac{(\lambda+\mu)\left(\lambda+\mu+\rho+\eta+f_{6}\left(e^{*}\right)\right)+p(\lambda+\mu+\rho)}{\left(\lambda+\mu+f_{5}\left(e^{*}\right)\right)\left(\lambda+\mu+\rho+\eta+f_{6}\left(e^{*}\right)\right)+p\left(\lambda+\mu+\rho+f_{6}\left(e^{*}\right)\right)} S^{*} f_{3}\left(e^{*}, \lambda\right) \\
& \quad+\frac{\left(\lambda+\mu+p+f_{5}\left(e^{*}\right)\right)(\lambda+\mu+\rho)+\eta(\lambda+\mu)}{\left(\lambda+\mu+p+f_{5}\left(e^{*}\right)\right)\left(\lambda+\mu+\rho+f_{6}\left(e^{*}\right)\right)+\eta\left(\lambda+\mu+f_{5}\left(e^{*}\right)\right)} V^{*} f_{4}\left(e^{*}, \lambda\right) \mid \\
& \quad<\left|S^{*} f_{3}\left(e^{*}, \lambda\right)+V^{*} f_{4}\left(e^{*}, \lambda\right)\right| \leq\left|S^{*} f_{3}\left(e^{*}, \lambda\right)\right|+\left|V^{*} f_{4}\left(e^{*}, \lambda\right)\right| \\
& \quad \leq S^{*} f_{5}\left(e^{*}\right)+V^{*} f_{6}\left(e^{*}\right)=1 .
\end{aligned}
$$

It follows from (5.6) that there is a contradiction. Therefore, $\operatorname{Re} \lambda<0$. This means that all the roots of (5.6) have negative real parts. Consequently, if $R_{0}>1$, the steady state $E^{*}$ is locally asymptotically stable. This completes the proof. 


\section{Uniform persistence}

In this section, we investigate the uniform persistence of system (1.5) by using the persistence theory for infinite dimensional dynamics system. Define

$$
\begin{aligned}
& \bar{a}=\inf \left\{a: \int_{a}^{\infty} \theta_{1}(u) \mathrm{d} u=0\right\}, \quad \bar{b}=\inf \left\{b: \int_{b}^{\infty} \theta_{2}(u) \mathrm{d} u=0\right\}, \\
& \bar{c}=\inf \left\{c: \int_{c}^{\infty} \theta_{3}(u) \mathrm{d} u=0\right\} .
\end{aligned}
$$

Since $\theta_{1}(a), \theta_{2}(a), \theta_{3}(a) \in L_{+}^{1}(0, \infty)$, we have $\bar{a}, \bar{b}, \bar{c}>0$. Furthermore, let

$$
\begin{aligned}
& \tilde{X}=L_{+}^{1}(0, \infty) \times L_{+}^{1}(0, \infty) \times L_{+}^{1}(0, \infty), \\
& \tilde{Y}=\left\{(e(t, \cdot), i(t, \cdot), j(t, \cdot))^{T} \in \tilde{X}: \int_{0}^{\bar{a}} e(t, x) \mathrm{d} x>0, \int_{0}^{\bar{b}} i(t, x) \mathrm{d} x>0, \int_{0}^{\bar{c}} j(t, x) \mathrm{d} x>0\right\},
\end{aligned}
$$

and

$$
Y=R^{+} \times R^{+} \times \tilde{Y}, \quad \partial Y=X \backslash Y, \quad \partial \tilde{Y}=\tilde{X} \backslash \tilde{Y} .
$$

It is not difficult to verify the following proposition.

Proposition 6.1 The subsets $Y$ and $\partial Y$ are both positively invariant under the semi-flow $\{\Phi(t)\}_{t \geq 0}$, namely, $\Phi(t, Y) \subset Y$ and $\Phi(t, \partial Y) \subset \partial Y$ for $t \geq 0$.

Furthermore, the following result is useful for the proof of uniform persistence.

Theorem 6.1 The disease-free steady state $E_{0}$ of system (1.5) is globally asymptotically stable for the semi-flow $\{\Phi(t)\}_{t \geq 0}$ restricted to $\partial Y$.

Proof Letting $\left(S_{0}, V_{0}, e_{0}(\cdot), i_{0}(\cdot), j_{0}(\cdot)\right) \in \partial Y$, namely, $\left(e_{0}(\cdot), i_{0}(\cdot), j_{0}(\cdot)\right) \in \partial \tilde{Y}$, we consider the following system:

$$
\begin{aligned}
& \frac{\partial e(t, a)}{\partial a}+\frac{\partial e(t, a)}{\partial t}=-\theta_{1}(a) e(t, a), \\
& \frac{\partial i(t, a)}{\partial a}+\frac{\partial i(t, a)}{\partial t}=-\theta_{2}(a) i(t, a), \\
& \frac{\partial j(t, a)}{\partial a}+\frac{\partial j(t, a)}{\partial t}=-\theta_{3}(a) j(t, a), \\
& e(t, 0)=S(t) \int_{0}^{\infty}\left(\frac{\beta_{1}(a) i(t, a)}{1+\alpha i(t, a)}+\beta_{3}(a) j(t, a)\right) \mathrm{d} a \\
& \quad+V(t) \int_{0}^{\infty}\left(\frac{\beta_{2}(a) i(t, a)}{1+\alpha i(t, a)}+\beta_{4}(a) j(t, a)\right) \mathrm{d} a, \\
& i(t, 0)=\int_{0}^{\infty} \gamma_{1}(a) e(t, a) \mathrm{d} a, \\
& j(t, 0)=\int_{0}^{\infty} \gamma_{2}(a) e(t, a) \mathrm{d} a+\int_{0}^{\infty} \xi(a) i(t, a) \mathrm{d} a, \\
& e(0, a)=\varphi_{e}(a), \quad i(0, a)=\varphi_{i}(a), \quad j(0, a)=\varphi_{j}(a) .
\end{aligned}
$$


Since $S(t) \leq S_{0}$ and $V(t) \leq V_{0}$ as $t$ tends to infinity, by comparison, we have $e(t, a) \leq$ $\tilde{e}(t, a), i(t, a) \leq \tilde{i}(t, a), j(t, a) \leq \tilde{j}(t, a)$, where $\tilde{e}(t, a), \tilde{i}(t, a)$ and $\tilde{j}(t, a)$ satisfy the following auxiliary system:

$$
\begin{aligned}
& \frac{\partial \tilde{e}(t, a)}{\partial a}+\frac{\partial \tilde{e}(t, a)}{\partial t}=-\theta_{1}(a) \tilde{e}(t, a), \\
& \frac{\partial \tilde{i}(t, a)}{\partial a}+\frac{\partial \tilde{i}(t, a)}{\partial t}=-\theta_{2}(a) \tilde{i}(t, a), \\
& \frac{\partial \tilde{j}(t, a)}{\partial a}+\frac{\partial \tilde{j}(t, a)}{\partial t}=-\theta_{3}(a) \tilde{j}(t, a), \\
& \tilde{e}(t, 0)=S_{0} \int_{0}^{\infty}\left(\frac{\beta_{1}(a) \tilde{i}(t, a)}{1+\alpha \tilde{i}(t, a)}+\beta_{3}(a) \tilde{j}(t, a)\right) \mathrm{d} a \\
& \quad+V_{0} \int_{0}^{\infty}\left(\frac{\beta_{2}(a) \tilde{i}(t, a)}{1+\alpha \tilde{i}(t, a)}+\beta_{4}(a) \tilde{j}(t, a)\right) \mathrm{d} a, \\
& \tilde{i}(t, 0)=\int_{0}^{\infty} \gamma_{1}(a) \tilde{e}(t, a) \mathrm{d} a, \\
& \tilde{j}(t, 0)=\int_{0}^{\infty} \gamma_{2}(a) \tilde{e}(t, a) \mathrm{d} a+\int_{0}^{\infty} \xi(a) \tilde{i}(t, a) \mathrm{d} a, \\
& \tilde{e}(0, a)=\varphi_{e}(a), \tilde{i}(0, a)=\varphi_{i}(a), \tilde{j}(0, a)=\varphi_{j}(a) .
\end{aligned}
$$

Similar to (2.2)-(2.4), solving the first three equations of (6.1) yields

$$
\begin{gathered}
\tilde{e}(t, a)= \begin{cases}\tilde{L}_{1}(t-a) B_{1}(a), & 0 \leq a \leq t, \\
\varphi_{e}(a-t) \frac{B_{1}(a)}{B_{1}(a-t)}, & 0 \leq t \leq a,\end{cases} \\
\tilde{i}(t, a)= \begin{cases}\tilde{L}_{2}(t-a) B_{2}(a), & 0 \leq a \leq t, \\
\varphi_{i}(a-t) \frac{B_{2}(a)}{B_{2}(a-t)}, & 0 \leq t \leq a,\end{cases} \\
\tilde{j}(t, a)= \begin{cases}\tilde{L}_{3}(t-a) B_{3}(a) & \text { for } 0 \leq a \leq t, \\
\varphi_{j}(a-t) \frac{B_{3}(a)}{B_{3}(a-t)} & \text { for } 0 \leq t \leq a,\end{cases}
\end{gathered}
$$

where

$$
\begin{aligned}
\tilde{L}_{1}(t)= & S_{0} \int_{0}^{\infty}\left(\frac{\beta_{1}(a) \tilde{i}(t, a)}{1+\alpha \tilde{i}(t, a)}+\beta_{3}(a) \tilde{j}(t, a)\right) \mathrm{d} a \\
& +V_{0} \int_{0}^{\infty}\left(\frac{\beta_{2}(a) \tilde{i}(t, a)}{1+\alpha \tilde{i}(t, a)}+\beta_{4}(a) \tilde{j}(t, a)\right) \mathrm{d} a \\
\tilde{L}_{2}(t)= & \int_{0}^{\infty} \gamma_{1}(a) \tilde{e}(t, a) \mathrm{d} a \\
\tilde{L}_{3}(t)= & \int_{0}^{\infty} \gamma_{2}(a) \tilde{e}(t, a) \mathrm{d} a+\int_{0}^{\infty} \xi(a) \tilde{i}(t, a) \mathrm{d} a .
\end{aligned}
$$


It follows from (6.2)-(6.4) that

$$
\begin{aligned}
\tilde{L}_{1}(t)= & S_{0} \int_{0}^{t}\left(\frac{\beta_{1}(a) \tilde{L}_{2}(t-a) B_{2}(a)}{1+\alpha \tilde{L}_{2}(t-a) B_{2}(a)}+\beta_{3}(a) \tilde{L}_{3}(t-a) B_{3}(a)\right) \mathrm{d} a \\
& +V_{0} \int_{0}^{t}\left(\frac{\beta_{2}(a) \tilde{L}_{2}(t-a) B_{2}(a)}{1+\alpha \tilde{L}_{2}(t-a) B_{2}(a)}+\beta_{4}(a) \tilde{L}_{3}(t-a) B_{3}(a)\right) \mathrm{d} a+G_{1}(t), \\
\tilde{L}_{2}(t)= & \int_{0}^{t} \gamma_{1}(a) \tilde{L}_{1}(t-a) B_{1}(a) \mathrm{d} a+G_{2}(t), \\
\tilde{L}_{3}(t)= & \int_{0}^{t} \gamma_{2}(a) \tilde{L}_{1}(t-a) B_{1}(a) \mathrm{d} a+\int_{0}^{t} \xi(a) \tilde{L}_{2}(t-a) B_{2}(a) \mathrm{d} a+G_{3}(t),
\end{aligned}
$$

where

$$
\begin{aligned}
G_{1}(t)= & S_{0} \int_{t}^{\infty}\left(\frac{\beta_{1}(a) \varphi_{i}(a-t) B_{2}(a)}{B_{2}(a-t)+\alpha \varphi_{i}(a-t) B_{2}(a)}+\beta_{3}(a) \varphi_{j}(a-t) \frac{B_{3}(a)}{B_{3}(a-t)}\right) \mathrm{d} a \\
& +V_{0} \int_{t}^{\infty}\left(\frac{\beta_{2}(a) \varphi_{i}(a-t) B_{2}(a)}{B_{2}(a-t)+\alpha \varphi_{i}(a-t) B_{2}(a)}+\beta_{4}(a) \varphi_{j}(a-t) \frac{B_{3}(a)}{B_{3}(a-t)}\right) \mathrm{d} a \\
G_{2}(t)= & \int_{t}^{\infty} \gamma_{1}(a) \varphi_{e}(a-t) \frac{B_{1}(a)}{B_{1}(a-t)} \mathrm{d} a \\
G_{3}(t)= & \int_{t}^{\infty} \gamma_{2}(a) \varphi_{e}(a-t) \frac{B_{1}(a)}{B_{1}(a-t)} \mathrm{d} a+\int_{t}^{\infty} \xi(a) \varphi_{i}(a-t) \frac{B_{2}(a)}{B_{2}(a-t)} \mathrm{d} a .
\end{aligned}
$$

Since $\left(\varphi_{e}(\cdot), \varphi_{i}(\cdot), \varphi_{j}(\cdot)\right) \in \partial \tilde{Y}$, we have $G_{i}(t) \equiv 0(i=1,2,3)$ for all $t \geq 0$. From (6.5), we obtain

$$
\begin{aligned}
\tilde{L}_{1}(t)= & S_{0} \int_{0}^{t}\left(\frac{\beta_{1}(a) \tilde{L}_{2}(t-a) B_{2}(a)}{1+\alpha \tilde{L}_{2}(t-a) B_{2}(a)}+\beta_{3}(a) \tilde{L}_{3}(t-a) B_{3}(a)\right) \mathrm{d} a \\
& +V_{0} \int_{0}^{t}\left(\frac{\beta_{2}(a) \tilde{L}_{2}(t-a) B_{2}(a)}{1+\alpha \tilde{L}_{2}(t-a) B_{2}(a)}+\beta_{4}(a) \tilde{L}_{3}(t-a) B_{3}(a)\right) \mathrm{d} a \\
\tilde{L}_{2}(t)= & \int_{0}^{t} \gamma_{1}(a) \tilde{L}_{1}(t-a) B_{1}(a) \mathrm{d} a \\
\tilde{L}_{3}(t)= & \int_{0}^{t} \gamma_{2}(a) \tilde{L}_{1}(t-a) B_{1}(a) \mathrm{d} a+\int_{0}^{t} \xi(a) \tilde{L}_{2}(t-a) B_{2}(a) \mathrm{d} a .
\end{aligned}
$$

It is easy to show that system (6.6) has a unique solution $\tilde{L}_{i}(t) \equiv 0(i=1,2,3)$. From (6.2)(6.4), we have $\tilde{e}(t, a)=0, \tilde{i}(t, a)=0, \tilde{j}(t, a)=0$. For $a \geq t$, it follows that

$$
\begin{aligned}
& \|\tilde{e}(t, a)\|_{L^{1}}=\left\|\varphi_{e}(a-t) \frac{B_{1}(a)}{B_{1}(a-t)}\right\|_{L^{1}} \leq e^{-\mu_{0} t}\left\|\varphi_{e}\right\|_{L^{1}}, \\
& \|\tilde{i}(t, a)\|_{L^{1}}=\left\|\varphi_{i}(a-t) \frac{B_{2}(a)}{B_{2}(a-t)}\right\|_{L^{1}} \leq e^{-\mu_{0} t}\left\|\varphi_{i}\right\|_{L^{1}}, \\
& \|\tilde{j}(t, a)\|_{L^{1}}=\left\|\varphi_{j}(a-t) \frac{B_{3}(a)}{B_{3}(a-t)}\right\|_{L^{1}} \leq e^{-\mu_{0} t}\left\|\varphi_{j}\right\|_{L^{1}},
\end{aligned}
$$

which implies that $\tilde{e}(t, a)=0, \tilde{i}(t, a)=0, \tilde{j}(t, a)=0$ as $t \rightarrow \infty$. Noting that $e(t, a) \leq \tilde{e}(t, a)$, $i(t, a) \leq \tilde{i}(t, a)$ and $j(t, a) \leq \tilde{j}(t, a)$, we have $e(t, a) \rightarrow 0, i(t, a) \rightarrow 0$ and $j(t, a) \rightarrow 0$ as $t \rightarrow \infty$. 
It follows from the first two equations of system (1.5) that $S(t) \rightarrow S_{0}$ and $V(t) \rightarrow V_{0}$ as $t \rightarrow \infty$. Thus, $E_{0}$ is globally asymptotically stable in $\partial Y$.

Theorem 6.2 If $R_{0}>1$, then the semi-flow $\{\Phi(t)\}_{t \geq 0}$ is uniformly persistent with respect to $(Y, \partial Y)$, i.e., there exists an $\varepsilon>0$ which is independent of initial values such that $\lim _{t \rightarrow \infty}\|\Phi(t, x)\|_{X} \geq \varepsilon$ for $x \in Y$. Furthermore, there is a compact subset $A_{0} \subset Y$ which is a global attractor for $\{\Phi(t, x)\}_{t \geq 0}$ in $Y$.

Proof It follows from Theorem 6.1 that $E_{0}$ is globally asymptotically stable in $\partial Y$. Applying Theorem 4.2 in [25], we need only to show that $W^{s}\left(E_{0}\right) \cap Y=\emptyset$, where

$$
W^{s}\left(E_{0}\right)=\left\{x \in Y: \lim _{t \rightarrow \infty} \Phi(t, x)=E_{0}\right\}
$$

Otherwise, there exists a solution $y \subset Y$ such that $\Phi(t, y) \rightarrow E_{0}$ as $t \rightarrow \infty$. In this case, there exists a sequence $\left\{y_{n}\right\} \subset Y$ such that $\left\|\Phi\left(t, y_{n}\right)-E_{0}\right\|_{X}<1 / n$ for $t \geq 0$. Denote $\Phi\left(t, y_{n}\right)=\left(S_{n}(t), V_{n}(t), e(t, \cdot), i(t, \cdot), j(t, \cdot)\right)$ and $y_{n}=\left(S_{n}(0), V_{n}(0), e(t, \cdot), i(t, \cdot), j(t, \cdot)\right)$. Since $R_{0}>1$, we can choose $n$ sufficiently large satisfying $S_{0}>1 / n$ and $V_{0}>1 / n$,

$$
\begin{aligned}
& \left(S_{0}-\frac{1}{n}\right) \int_{0}^{\infty}\left[K_{1} K_{3}(a)+\left(K_{2}+K_{1} K_{7}\right) K_{4}(a)\right] \mathrm{d} a \\
& \quad+\left(V_{0}-\frac{1}{n}\right) \int_{0}^{\infty}\left[K_{1} K_{5}(a)+\left(K_{2}+K_{1} K_{7}\right) K_{6}(a)\right] \mathrm{d} a>1 .
\end{aligned}
$$

For such a $n>0$, there exists a $T>0$ such that, for $t>T, S_{0}-1 / n<S_{n}(t)<S_{0}+1 / n$ and $V_{0}-1 / n<V_{n}(t)<V_{0}+1 / n$. Consider the following auxiliary system:

$$
\begin{aligned}
& \frac{\partial \hat{e}(t, a)}{\partial a}+\frac{\partial \hat{e}(t, a)}{\partial t}=-\theta_{1}(a) \hat{e}(t, a), \\
& \frac{\partial \hat{i}(t, a)}{\partial a}+\frac{\partial \hat{i}(t, a)}{\partial t}=-\theta_{2}(a) \hat{i}(t, a), \\
& \frac{\partial \hat{j}(t, a)}{\partial a}+\frac{\partial \hat{j}(t, a)}{\partial t}=-\theta_{3}(a) \hat{j}(t, a), \\
& \hat{e}(t, 0)=\left(S_{0}-\frac{1}{n}\right) \int_{0}^{\infty}\left(\frac{\beta_{1}(a) \hat{i}(t, a)}{1+\alpha \hat{i}(t, a)}+\beta_{3}(a) \hat{j}(t, a)\right) \mathrm{d} a \\
& \quad+\left(V_{0}-\frac{1}{n}\right) \int_{0}^{\infty}\left(\frac{\beta_{2}(a) \hat{i}(t, a)}{1+\alpha \hat{i}(t, a)}+\beta_{4}(a) \hat{j}(t, a)\right) \mathrm{d} a, \\
& \hat{i}(t, 0)=\int_{0}^{\infty} \gamma_{1}(a) \hat{e}(t, a) \mathrm{d} a, \\
& \hat{j}(t, 0)=\int_{0}^{\infty} \gamma_{2}(a) \hat{e}(t, a) \mathrm{d} a+\int_{0}^{\infty} \xi(a) \hat{i}(t, a) \mathrm{d} a .
\end{aligned}
$$

Looking for solutions of system (6.8) of the form $\hat{e}(t, a)=\hat{e}(a) e^{\lambda t}, \hat{i}(t, a)=\hat{i}(a) e^{\lambda t}$ and $\hat{j}(t, a)=\hat{j}(a) e^{\lambda t}$, where the functions $\hat{e}(a), \hat{i}(a)$ and $\hat{j}(a)$ will be determined later, we obtain the following linear eigenvalue problem:

$$
\frac{\partial \hat{e}(a)}{\partial a}=-\left(\lambda+\theta_{1}(a)\right) \hat{e}(a)
$$




$$
\begin{aligned}
\frac{\partial \hat{i}(a)}{\partial a}= & -\left(\lambda+\theta_{2}(a)\right) \hat{i}(a), \\
\frac{\partial \hat{j}(a)}{\partial a}= & -\left(\lambda+\theta_{3}(a)\right) \hat{j}(a), \\
\hat{e}(0)= & \left(S_{0}-\frac{1}{n}\right) \int_{0}^{\infty}\left(\frac{\beta_{1}(a) \hat{i}(a)}{1+\alpha \hat{i}(a) e^{\lambda t}}+\beta_{3}(a) \hat{j}(a)\right) \mathrm{d} a \\
& +\left(V_{0}-\frac{1}{n}\right) \int_{0}^{\infty}\left(\frac{\beta_{2}(a) \hat{i}(a)}{1+\alpha \hat{i}(a) e^{\lambda t}}+\beta_{4}(a) \hat{j}(a)\right) \mathrm{d} a, \\
\hat{i}(0)= & \int_{0}^{\infty} \gamma_{1}(a) \hat{e}(a) \mathrm{d} a, \\
\hat{j}(0)= & \int_{0}^{\infty} \gamma_{2}(a) \hat{e}(a) \mathrm{d} a+\int_{0}^{\infty} \xi(a) \hat{i}(a) \mathrm{d} a .
\end{aligned}
$$

Solving the first three equations of system (6.9) yields

$$
\begin{aligned}
& \hat{e}(a)=\hat{e}(0) \exp \left[-\int_{0}^{a}\left(\lambda+\theta_{1}(s)\right) \mathrm{d} s\right], \quad \hat{i}(a)=\hat{i}(0) \exp \left[-\int_{0}^{a}\left(\lambda+\theta_{2}(s)\right) \mathrm{d} s\right], \\
& \hat{j}(a)=\hat{j}(0) \exp \left[-\int_{0}^{a}\left(\lambda+\theta_{3}(s)\right) \mathrm{d} s\right] .
\end{aligned}
$$

Substituting (6.10) into the last three equations of (6.9), we obtain the characteristic equation of system (6.8) at the steady state $E_{0}$ as follows:

$$
f(\lambda)=1,
$$

where

$$
\begin{aligned}
f(\lambda)= & \left(S_{0}-\frac{1}{n}\right) \\
& \times \int_{0}^{\infty} \frac{\beta_{1}(a) \int_{0}^{\infty} \gamma_{1}(a) \exp \left[-\int_{0}^{a}\left(\lambda+\theta_{1}(s)\right) \mathrm{d} s\right] \mathrm{d} a \exp \left[-\int_{0}^{a}\left(\lambda+\theta_{2}(s)\right) \mathrm{d} s\right]}{1+\alpha \hat{i}(0) \exp \left[\lambda t-\int_{0}^{a}\left(\lambda+\theta_{2}(s)\right) \mathrm{d} s\right]} \mathrm{d} a \\
& +\left(S_{0}-\frac{1}{n}\right) \int_{0}^{\infty}\left\{\beta_{3}(a) \exp \left[-\int_{0}^{a}\left(\lambda+\theta_{3}(s)\right) \mathrm{d} s\right]\right. \\
& \left.\times \int_{0}^{\infty} \gamma_{2}(a) \exp \left[-\int_{0}^{a}\left(\lambda+\theta_{1}(s)\right) \mathrm{d} s\right] \mathrm{d} a\right\} \mathrm{d} a \\
& +\left(S_{0}-\frac{1}{n}\right) \int_{0}^{\infty} \beta_{3}(a) \exp \left[-\int_{0}^{a}\left(\lambda+\theta_{3}(s)\right) \mathrm{d} s\right] \\
& \times \int_{0}^{\infty} \xi(a) \exp \left[-\int_{0}^{a}\left(\lambda+\theta_{2}(s)\right) \mathrm{d} s\right] \\
& \times \int_{0}^{\infty} \gamma_{1}(a) \exp \left[-\int_{0}^{a}\left(\lambda+\theta_{1}(s)\right) \mathrm{d} s\right] \mathrm{d} a \mathrm{~d} a \mathrm{~d} a \\
& +\left(V_{0}-\frac{1}{n}\right) \\
& \times \int_{0}^{\infty} \frac{\beta_{2}(a) \exp \left[-\int_{0}^{a}\left(\lambda+\theta_{2}(s)\right) \mathrm{d} s\right] \int_{0}^{\infty} \gamma_{1}(a) \exp \left[-\int_{0}^{a}\left(\lambda+\theta_{1}(s)\right) \mathrm{d} s\right] \mathrm{d} a}{1+\alpha \hat{i}(0) \exp \left[\lambda t-\int_{0}^{a}\left(\lambda+\theta_{2}(s)\right) \mathrm{d} s\right]}
\end{aligned}
$$




$$
\begin{aligned}
& +\left(V_{0}-\frac{1}{n}\right) \int_{0}^{\infty}\left\{\beta_{4}(a) \exp \left[-\int_{0}^{a}\left(\lambda+\theta_{3}(s)\right) \mathrm{d} s\right]\right. \\
& \left.\times \int_{0}^{\infty} \gamma_{2}(a) \exp \left[-\int_{0}^{a}\left(\lambda+\theta_{1}(s)\right) \mathrm{d} s\right] \mathrm{d} a\right\} \mathrm{d} a \\
& +\left(V_{0}-\frac{1}{n}\right) \int_{0}^{\infty} \beta_{4}(a) \exp \left[-\int_{0}^{a}\left(\lambda+\theta_{3}(s)\right) \mathrm{d} s\right] \\
& \times \int_{0}^{\infty} \xi(a) \exp \left[-\int_{0}^{a}\left(\lambda+\theta_{2}(s)\right) \mathrm{d} s\right] \\
& \times \int_{0}^{\infty} \gamma_{1}(a) \exp \left[-\int_{0}^{a}\left(\lambda+\theta_{1}(s)\right) \mathrm{d} s\right] \mathrm{d} a \mathrm{~d} a \mathrm{~d} a .
\end{aligned}
$$

Clearly, we have $\lim _{\lambda \rightarrow \infty} f(\lambda)=0$ and

$$
\begin{aligned}
f(0)< & \left(S_{0}-\frac{1}{n}\right) \int_{0}^{\infty}\left[K_{1} K_{3}(a)+\left(K_{2}+K_{1} K_{7}\right) K_{4}(a)\right] \mathrm{d} a \\
& +\left(V_{0}-\frac{1}{n}\right) \int_{0}^{\infty}\left[K_{1} K_{5}(a)+\left(K_{2}+K_{1} K_{7}\right) K_{6}(a)\right] \mathrm{d} a .
\end{aligned}
$$

From (6.7), there exist a $n>0$ and a $T>0$ such that

$$
\begin{aligned}
& \left(S_{0}-\frac{1}{n}\right) \int_{0}^{\infty}\left[K_{1} K_{3}(a)+\left(K_{2}+K_{1} K_{7}\right) K_{4}(a)\right] \mathrm{d} a \\
& \quad+\left(V_{0}-\frac{1}{n}\right) \int_{0}^{\infty}\left[K_{1} K_{5}(a)+\left(K_{2}+K_{1} K_{7}\right) K_{6}(a)\right] \mathrm{d} a>1 .
\end{aligned}
$$

Hence, if $R_{0}>1$, Eq. (6.11) has at least one positive root. This implies that the solution $(\hat{e}(t, \cdot), \hat{i}(t, \cdot), \hat{j}(t, \cdot))$ of system (6.8) is unbounded. By comparison, the solution $\Phi\left(t, y_{n}\right)$ of system (1.5) is unbounded, which contradicts Proposition 2.1. Therefore, the semi-flow $\Phi(t)_{t \geq 0}$ generated by system (1.5) is uniformly persistent. Furthermore, there is a compact subset $A_{0} \subset Y$ which is a global attractor for $\Phi(t)_{t \geq 0}$ in $Y$. This completes the proof.

\section{Global stability}

This section is devoted to the global stability of equilibria. Before going into details, we make some preparations.

First, we introduce an important function which is obtained from the linear combination of Volterra-type functions of the form

$$
g(x)=x-1-\ln x
$$

Obviously, $g(x) \geq 0$ for $x>0$ and $g^{\prime}(x)=1-1 / x$. Then $g(x)$ has a global minimum at $x=1$ and $g(1)=0$.

Theorem 7.1 If $R_{0}<1$, the disease-free steady state $E_{0}$ is globally asymptotically stable.

Proof Define a Lyapunov functional as

$$
V_{1}=V_{11}+V_{12}+V_{13}
$$


where

$$
\begin{aligned}
& V_{11}=S_{0} g\left(S(t) / S_{0}\right)+V_{0} g\left(V(t) / V_{0}\right), \quad V_{12}=\int_{0}^{\infty} \omega_{1}(a) e(t, a) \mathrm{d} a, \\
& V_{13}=\int_{0}^{\infty} \omega_{2}(a) i(t, a) \mathrm{d} a, \quad V_{14}=\int_{0}^{\infty} \omega_{3}(a) j(t, a) \mathrm{d} a,
\end{aligned}
$$

where

$$
\begin{aligned}
& \omega_{1}(a)=\int_{a}^{\infty}\left(\omega_{3}(0) \gamma_{2}(x)+\omega_{2}(0) \gamma_{1}(x)\right) \exp \left(-\int_{a}^{x} \theta_{1}(\tau) d \tau\right) d x \\
& \omega_{2}(a)=\int_{a}^{\infty}\left(S_{0} \beta_{1}(x)+V_{0} \beta_{2}(x)+\omega_{3}(0) \xi(x)\right) \exp \left(-\int_{a}^{x} \theta_{2}(\tau) d \tau\right) d x \\
& \omega_{3}(a)=\int_{a}^{\infty}\left(S_{0} \beta_{3}(x)+V_{0} \beta_{4}(x)\right) \exp \left(-\int_{a}^{x} \theta_{3}(\tau) d \tau\right) d x
\end{aligned}
$$

then

$$
\begin{aligned}
& \omega_{2}(0) \gamma_{1}(a)+\omega_{3}(0) \gamma_{2}(a)+\omega_{1}^{\prime}(a)-\theta_{1}(a) \omega_{1}(a)=0, \\
& S_{0} \beta_{1}(a)+V_{0} \beta_{2}+\omega_{3}(0) \xi(a)+\omega_{2}^{\prime}(a)-\theta_{2}(a) \omega_{2}(a)=0, \\
& S_{0} \beta_{3}(a)+V_{0} \beta_{4}+\omega_{3}^{\prime}(a)-\theta_{3}(a) \omega_{3}(a)=0 .
\end{aligned}
$$

The derivative of $V_{11}$ along with the solution of system (1.5) can be calculated as

$$
\begin{aligned}
\frac{\mathrm{d} V_{11}}{\mathrm{~d} t}= & \left(1-\frac{S_{0}}{S(t)}\right)\left(\Lambda-(\mu+p) S(t)+\eta V(t)-S(t) \int_{0}^{\infty} \frac{\beta_{1}(a) i(t, a)}{1+\alpha i(t, a)} \mathrm{d} a\right. \\
& \left.+S(t) \int_{0}^{\infty} \beta_{3}(a) j(t, a) \mathrm{d} a\right) \\
& +\left(1-\frac{V_{0}}{V(t)}\right)\left(p S(t)-(\mu+\rho+\eta) V(t)-V(t) \int_{0}^{\infty} \frac{\beta_{2}(a) i(t, a)}{1+\alpha i(t, a)} \mathrm{d} a\right. \\
& \left.-V(t) \int_{0}^{\infty} \beta_{4}(a) j(t, a) \mathrm{d} a\right) \\
= & \mu S_{0}\left(2-\frac{S(t)}{S_{0}}-\frac{S_{0}}{S(t)}\right)+(\mu+\rho) V_{0}\left(3-\frac{V(t)}{V_{0}}-\frac{S_{0}}{S(t)}-\frac{S(t) V_{0}}{S_{0} V(t)}\right) \\
& +\eta V_{0}\left(2-\frac{S(t) V_{0}}{S_{0} V(t)}-\frac{S_{0} V(t)}{S(t) V_{0}}\right) \\
& -\left(S(t)-S_{0}\right) \int_{0}^{\infty}\left(\frac{\beta_{1}(a) i(t, a)}{1+\alpha i(t, a)}+\beta_{3}(a) j(t, a)\right) \mathrm{d} a \\
& -\left(V(t)-V_{0}\right) \int_{0}^{\infty}\left(\frac{\beta_{2}(a) i(t, a)}{1+\alpha i(t, a)}+\beta_{4}(a) j(t, a)\right) \mathrm{d} a .
\end{aligned}
$$

The derivative of $V_{12}$ along with the solution of system (1.5) can be calculated as

$$
\begin{aligned}
\frac{d V_{12}}{d t}= & \frac{d}{d t} \int_{0}^{t} \omega_{1}(a) e(t-a, 0) \exp \left(-\int_{0}^{a} \theta_{1}(\tau) d \tau\right) \mathrm{d} a \\
& +\frac{d}{d t} \int_{t}^{\infty} \omega_{1}(a) \varphi_{e}(a-t) \exp \left(-\int_{a-t}^{a} \theta_{1}(\tau) d \tau\right) \mathrm{d} a .
\end{aligned}
$$


Let $r=t-a$, then

$$
\begin{aligned}
\frac{\mathrm{d} V_{12}}{\mathrm{~d} t}= & \frac{\mathrm{d}}{\mathrm{d} t} \int_{0}^{t} \omega_{1}(t-r) e(r, 0) \exp \left(-\int_{0}^{t-r} \theta_{1}(\tau) d \tau\right) \mathrm{d} r \\
& +\frac{\mathrm{d}}{\mathrm{d} t} \int_{t}^{\infty} \omega_{1}(t+r) \varphi_{e}(r) \exp \left(-\int_{r}^{t+r} \theta_{1}(\tau) d \tau\right) \mathrm{d} r \\
= & \omega_{1}(0) e(t, 0)+\int_{0}^{\infty}\left(\omega^{\prime}(a)-\theta_{1}(a) \omega_{1}(a)\right) e(t, a) \mathrm{d} a .
\end{aligned}
$$

Similarly, we can get

$$
\begin{aligned}
\frac{\mathrm{d} V_{13}}{\mathrm{~d} t}= & \omega_{2}(0) \int_{0}^{\infty} \gamma_{1}(a) e(t, a) \mathrm{d} a+\int_{0}^{\infty}\left(\omega_{2}^{\prime}(a)-\theta_{2}(a) \omega_{2}(a)\right) i(t, a) \mathrm{d} a, \\
\frac{\mathrm{d} V_{14}}{\mathrm{~d} t}= & \omega_{3}(0) \int_{0}^{\infty}\left(\gamma_{2}(a) e(t, a)+\xi(a) i(t, a)\right) \mathrm{d} a \\
& +\int_{0}^{\infty}\left(\omega_{3}^{\prime}(a)-\theta_{3}(a) \omega_{3}(a)\right) j(t, a) \mathrm{d} a .
\end{aligned}
$$

Combining the (7.1)-(7.3), it is easy to get

$$
\begin{aligned}
\frac{\mathrm{d} V_{1}}{\mathrm{~d} t}= & \mu S_{0}\left(2-\frac{S_{0}}{S(t)}-\frac{S(t)}{S_{0}}\right)+(\mu+\rho) V_{0}\left(3-\frac{V(t)}{V_{0}}-\frac{S_{0}}{S(t)}-\frac{S(t) V_{0}}{S_{0} V(t)}\right) \\
& +\eta V_{0}\left(2-\frac{S(t) V_{0}}{S_{0} V(t)}-\frac{S_{0} V(t)}{S(t) V_{0}}\right)-e(t, 0)+\omega_{1}(0) e(t, 0) \\
& +\left(S_{0} \frac{\beta_{1}(a)}{1+\alpha i(t, a)}+V_{0} \frac{\beta_{2}(a)}{1+\alpha i(t, a)}+\omega_{2}(0) \xi(a)+\omega_{1}^{\prime}(a)-\theta_{1}(a) \omega_{1}(a)\right) i(t, a) \mathrm{d} a \\
& +\int_{0}^{\infty}\left(S_{0} \beta_{3}(a)+V_{0} \beta_{4}(a)+\omega_{3}^{\prime}(a)-\theta_{3}(a) \omega_{3}(a)\right) j(t, a) \mathrm{d} a \\
& +\int_{0}^{\infty}\left(\omega_{2}(0) \gamma_{1}(a)+\omega_{3}(0) \gamma_{2}(a)+\omega^{\prime}(a)-\theta_{1}(a) \omega_{1}(a)\right) e(t, a) \mathrm{d} a \\
\leq & \mu S_{0}\left(2-\frac{S_{0}}{S(t)}-\frac{S(t)}{S_{0}}\right)+(\mu+\rho) V_{0}\left(3-\frac{V(t)}{V_{0}}-\frac{S_{0}}{S(t)}-\frac{S(t) V_{0}}{S_{0} V(t)}\right) \\
& +\eta V_{0}\left(2-\frac{S(t) V_{0}}{S_{0} V(t)}-\frac{S_{0} V(t)}{S(t) V_{0}}\right)+\left(R_{0}-1\right) e(t, 0) .
\end{aligned}
$$

Therefore, $R_{0} \leq 1$ ensures that $\mathrm{d} V_{1} / \mathrm{d} t \leq 0$ holds. Furthermore, the strict equality holds if and only if $S=S_{0}, V=V_{0}, e(t, a)=0, i(t, a)=0$ and $j(t, a)=0$, simultaneously. Thus, $M_{0}=E_{0} \subset \Upsilon$ is the largest invariant subset of $\mathrm{d} V_{1} / \mathrm{d} t=0$, and by the Lyapunov-LaSalle invariance principle, the steady state $E_{0}$ is globally asymptotically stable when $R_{0} \leq 1$.

Theorem 7.2 If $R_{0}>1$, the steady state $E^{*}$ is globally asymptotically stable.

Proof Constructing a Lyapunov functional as follows

$$
V_{2}=V_{21}+V_{22}+V_{23}+V_{24}
$$


where

$$
\begin{aligned}
V_{21}= & g\left(S(t) / S^{*}\right)+g\left(V(t) / V^{*}\right), \\
V_{22}= & \int_{0}^{\infty} \frac{S^{*} \beta_{1}(a) B_{2}(a)+V^{*} \beta_{2}(a) B_{2}(a)}{1+\alpha i^{*}(a)} \mathrm{d} a \int_{0}^{\infty} q_{1}(a) g\left(e(t, a) / e^{*}(a)\right) \mathrm{d} a \\
& +\int_{0}^{\infty}\left(S^{*} \beta_{3}(a) B_{3}(a)+V^{*} \beta_{4}(a) B_{3}(a)\right) \mathrm{d} a\left(\int_{0}^{\infty} q_{2}(a) g\left(e(t, a) / e^{*}(a)\right) \mathrm{d} a\right. \\
& \left.+\int_{0}^{\infty} q_{3}(a) g\left(i(t, a) / i^{*}(a)\right) \mathrm{d} a\right), \\
V_{23}= & \int_{0}^{\infty} q_{i}(a) g\left(i(t, a)\left(1+\alpha i^{*}(a)\right) / i^{*}(a)(1+\alpha i(t, a))\right) \mathrm{d} a, \\
V_{24}= & \int_{0}^{\infty} q_{j}(a) g\left(j(t, a) / j^{*}(a)\right) \mathrm{d} a,
\end{aligned}
$$

where

$$
\begin{aligned}
& q_{1}(a)=\int_{a}^{\infty} \gamma_{1}(\sigma) e^{*}(\sigma) \mathrm{d} \sigma, \quad q_{2}(a)=\int_{a}^{\infty} \gamma_{2}(\sigma) e^{*}(\sigma) \mathrm{d} \sigma \\
& q_{3}(a)=\int_{a}^{\infty} \xi(\sigma) i^{*}(\sigma) \mathrm{d} \sigma, \quad q_{i}(a)=\int_{a}^{\infty} \frac{\beta_{1}(\sigma) S^{*}+\beta_{2}(\sigma) V^{*}}{1+\alpha i^{*}(\sigma)} i^{*}(\sigma) \mathrm{d} \sigma, \\
& q_{j}(a)=\int_{a}^{\infty}\left(\beta_{3}(\sigma) S^{*}+\beta_{4}(\sigma) V^{*}\right) j^{*}(\sigma) \mathrm{d} \sigma .
\end{aligned}
$$

Calculating the derivative of $V_{21}$ along with the solution of system (1.5), we have

$$
\begin{aligned}
\frac{\mathrm{d} V_{21}}{\mathrm{~d} t}= & \left(1-\frac{S^{*}}{S(t)}\right)(\Lambda-(\mu+p) S(t)+\eta V(t) \\
& \left.-S(t) \int_{0}^{\infty}\left(\frac{\beta_{1}(a) i(t, a)}{1+\alpha i(t, a)}+\beta_{3}(a) j(t, a)\right) \mathrm{d} a\right) \\
& +\left(1-\frac{V^{*}}{V(t)}\right)\left(p S(t)-(\mu+\rho+\eta) V(t)-V(t) \int_{0}^{\infty} \frac{\beta_{2}(a) i(t, a)}{1+\alpha i(t, a)} \mathrm{d} a\right. \\
& \left.-V(t) \int_{0}^{\infty} \beta_{4}(a) j(t, a) \mathrm{d} a\right) \\
= & \mu S^{*}\left(2-\frac{S(t)}{S^{*}}-\frac{S^{*}}{S(t)}\right)+\left(\mu+\rho+\int_{0}^{\infty}\left(\frac{\beta_{2}(a) i^{*}(a)}{1+\alpha i^{*}(a)}+\beta_{4}(a) j^{*}(a)\right) \mathrm{d} a\right) V^{*} \\
& \times\left(3-\frac{V(t)}{V^{*}}-\frac{S^{*}}{S(t)}-\frac{S(t) V^{*}}{S^{*} V(t)}\right)+\eta V^{*}\left(2-\frac{S^{*} V(t)}{S(t) V^{*}}-\frac{S(t) V^{*}}{S^{*} V(t)}\right) \\
& +S^{*} \int_{0}^{\infty}\left(\frac{\beta_{1}(a) i^{*}(a)}{1+\alpha i^{*}(a)}+\beta_{3}(a) j^{*}(a)\right) \mathrm{d} a \\
& -S(t) \int_{0}^{\infty}\left(\frac{\beta_{1}(a) i(t, a)}{1+\alpha i(t, a)}+\beta_{3}(a) j(t, a)\right) \mathrm{d} a \\
& -\frac{S^{*}}{S(t)} S^{*} \int_{0}^{\infty}\left(\frac{\beta_{1}(a) i^{*}(a)}{1+\alpha i^{*}(a)}+\beta_{3}(a) j^{*}(a)\right) \mathrm{d} a \\
& +S^{*} \int_{0}^{\infty}\left(\frac{\beta_{1}(a) i(t, a)}{1+\alpha i(t, a)}+\beta_{3}(a) j(t, a)\right) \mathrm{d} a-V^{*} \int_{0}^{\infty}\left(\frac{\beta_{2} i^{*}(a)}{1+\alpha i^{*}(a)}\right.
\end{aligned}
$$




$$
\begin{aligned}
& \left.+\beta_{4}(a) j^{*}(a)\right) \mathrm{d} a+V^{*} \int_{0}^{\infty}\left(\frac{\beta_{2}(a) i(t, a)}{1+\alpha i(t, a)}+\beta_{4}(a) j(t, a)\right) \mathrm{d} a \\
& -V(t) \int_{0}^{\infty}\left(\frac{\beta_{2}(a) i(t, a)}{1+\alpha i(t, a)}+\beta_{4}(a) j(t, a)\right) \mathrm{d} a \\
& +V(t) \int_{0}^{\infty}\left(\frac{\beta_{2}(a) i^{*}(a)}{1+\alpha i^{*}(a)}+\beta_{4}(a) j^{*}(a)\right) \mathrm{d} a
\end{aligned}
$$

It follows that

$$
\begin{aligned}
\frac{\mathrm{d} V_{21}}{\mathrm{~d} t}= & \mu S^{*}\left(2-\frac{S(t)}{S^{*}}-\frac{S^{*}}{S(t)}\right)+\left(\mu+\rho+\int_{0}^{\infty}\left(\frac{\beta_{2}(a) i^{*}(a)}{1+\alpha i^{*}(a)}\right.\right. \\
& \left.\left.+\beta_{4}(a) j^{*}(a)\right) \mathrm{d} a\right) V^{*}\left(3-\frac{V(t)}{V^{*}}-\frac{S^{*}}{S(t)}-\frac{S(t) V^{*}}{S^{*} V(t)}\right) \\
& +\eta V^{*}\left(2-\frac{S^{*} V(t)}{S(t) V^{*}}-\frac{S(t) V^{*}}{S^{*} V(t)}\right)+\int_{0}^{\infty}\left(\frac{\beta_{1}(a) S^{*} i^{*}(a)}{1+\alpha i^{*}(a)}+\beta_{3}(a) S^{*} j^{*}(a)\right) \mathrm{d} a \\
& -\frac{S(t)}{S^{*}} \int_{0}^{\infty}\left(\frac{\beta_{1}(a) S^{*} i(t, a)}{1+\alpha i(t, a)}+\beta_{3}(a) S^{*} j(t, a)\right) \mathrm{d} a \\
& -\frac{S^{*}}{S(t)} \int_{0}^{\infty}\left(\frac{\beta_{1}(a) S^{*} i^{*}(a)}{1+\alpha i^{*}(a)}+\beta_{3}(a) S^{*} j^{*}(a)\right) \mathrm{d} a \\
& +\int_{0}^{\infty}\left(\frac{\beta_{1}(a) S^{*} i(t, a)}{1+\alpha i(t, a)}+\beta_{3}(a) S^{*} j(t, a)\right) \mathrm{d} a-\int_{0}^{\infty}\left(\frac{\beta_{2}(a) V^{*} i^{*}(a)}{1+\alpha i^{*}(a)}\right. \\
& \left.+\beta_{4}(a) V^{*} j^{*}(a)\right) \mathrm{d} a+\int_{0}^{\infty}\left(\frac{\beta_{2}(a) V^{*} i(t, a)}{1+\alpha i^{*}(a)}+\beta_{4}(a) V^{*} j(t, a)\right) \mathrm{d} a \\
& -\frac{V(t)}{V^{*}} \int_{0}^{\infty}\left(\frac{\beta_{2}(a) V^{*} i(t, a)}{1+\alpha i(t, a)}+\beta_{4}(a) V^{*} j(t, a)\right) \mathrm{d} a \\
& +\frac{V(t)}{V^{*}} \int_{0}^{\infty}\left(\frac{\beta_{2}(a) V^{*} i^{*}(a)}{1+\alpha i^{*}(a)}+\beta_{4}(a) V^{*} j^{*}(a)\right) \mathrm{d} a .
\end{aligned}
$$

The derivative of $V_{22}, V_{23}$ and $V_{24}$ can be calculated as follows:

$$
\begin{aligned}
\frac{\mathrm{d} V_{22}}{\mathrm{~d} t}= & \int_{0}^{\infty} \frac{S^{*} \beta_{1}(a) B_{2}(a)+V^{*} \beta_{2}(a) B_{2}(a)}{1+\alpha i^{*}(a)} \mathrm{d} a \int_{0}^{\infty} \gamma_{1}(a) e^{*}(a)\left\{\frac{e(t, 0)}{e^{*}(0)}-\frac{e(t, a)}{e^{*}(a)}\right. \\
& \left.+\ln \frac{e(t, a)}{e^{*}(a)}-\ln \frac{e(t, 0)}{e^{*}(0)}\right\} \mathrm{d} a \\
& +\int_{0}^{\infty}\left(S^{*} \beta_{3}(a) B_{3}(a)+V^{*} \beta_{4}(a) B_{3}(a)\right) \mathrm{d} a \int_{0}^{\infty} \gamma_{2}(a) e^{*}(a)\left\{\frac{e(t, 0)}{e^{*}(0)}-\frac{e(t, a)}{e^{*}(a)}\right. \\
& \left.+\ln \frac{e(t, a)}{e^{*}(a)}-\ln \frac{e(t, 0)}{e^{*}(0)}\right\} \mathrm{d} a \\
& +\int_{0}^{\infty}\left(S^{*} \beta_{3}(a) B_{3}(a)+V^{*} \beta_{4}(a) B_{3}(a)\right) \mathrm{d} a \int_{0}^{\infty} \xi(a) i^{*}(a)\left\{\frac{i(t, 0)}{i^{*}(0)}-\frac{i(t, a)}{i^{*}(a)}\right. \\
& \left.+\ln \frac{i(t, a)}{i^{*}(a)}-\ln \frac{i(t, 0)}{i^{*}(0)}\right\} \mathrm{d} a, \\
\frac{\mathrm{d} V_{23}}{\mathrm{~d} t}= & \int_{0}^{\infty} \frac{\left(\beta_{1}(a) S^{*}+\beta_{2}(a) V^{*}\right) i^{*}(a)}{1+\alpha i^{*}(a)}\left\{\frac{i(t, 0)\left(1+\alpha i^{*}(0)\right)}{i^{*}(0)(1+\alpha i(t, 0))}-\frac{i(t, a)\left(1+\alpha i^{*}(a)\right)}{i^{*}(a)(1+\alpha i(t, a))}\right. \\
& \left.+\ln \frac{i(t, a)\left(1+\alpha i^{*}(a)\right)}{i^{*}(a)(1+\alpha i(t, a))}-\ln \frac{i(t, 0)\left(1+\alpha i^{*}(0)\right)}{i^{*}(0)(1+\alpha i(t, 0))}\right\} \mathrm{d} a,
\end{aligned}
$$




$$
\frac{\mathrm{d} V_{24}}{\mathrm{~d} t}=\int_{0}^{\infty}\left(\beta_{3}(a) S^{*}+\beta_{4}(a) V^{*}\right) j^{*}(a)\left\{\frac{j(t, 0)}{j^{*}(0)}-\frac{j(t, a)}{j^{*}(a)}+\ln \frac{j(t, a)}{j^{*}(a)}-\ln \frac{j(t, 0)}{j^{*}(0)}\right\} \mathrm{d} a
$$

\section{Combining (7.4)-(7.7), we get}

$$
\begin{aligned}
& \frac{\mathrm{d} V_{2}}{\mathrm{~d} t}=\mu S^{*}\left(2-\frac{S(t)}{S^{*}}-\frac{S^{*}}{S(t)}\right)+\left(\mu+\rho+\int_{0}^{\infty}\left(\frac{\beta_{2}(a) i^{*}(a)}{1+\alpha i^{*}(a)}+\beta_{4}(a) j^{*}(a)\right) \mathrm{d} a\right) V^{*} \\
& \times\left(3-\frac{V(t)}{V^{*}}-\frac{S^{*}}{S(t)}-\frac{S(t) V^{*}}{S^{*} V(t)}\right)+\eta V^{*}\left(2-\frac{S^{*} V(t)}{S(t) V^{*}}-\frac{S(t) V^{*}}{S^{*} V(t)}\right) \\
& +\int_{0}^{\infty} \frac{\beta_{1}(a) S^{*} i^{*}(a)}{1+\alpha i^{*}(a)}\left\{1-\frac{S(t) i(t, a)\left(1+\alpha i^{*}(a)\right)}{S^{*} i^{*}(a)(1+\alpha i(t, a))}-\frac{S^{*}}{S(t)}+\frac{i(t, 0)\left(1+\alpha i^{*}(0)\right)}{i^{*}(0)(1+\alpha i(t, 0))}\right. \\
& \left.+\ln \frac{i(t, a)\left(1+\alpha i^{*}(a)\right)}{i^{*}(a)(1+\alpha i(t, a))}-\ln \frac{i(t, 0)\left(1+\alpha i^{*}(0)\right)}{i^{*}(0)(1+\alpha i(t, 0))}\right\} \mathrm{d} a \\
& +\int_{0}^{\infty} \frac{\beta_{2}(a) V^{*} i^{*}(a)}{1+\alpha i^{*}(a)}\left\{-1-\frac{V(t) i(t, a)\left(1+\alpha i^{*}(a)\right)}{V^{*} i^{*}(a)(1+\alpha i(t, a))}+\frac{V^{*}}{V(t)}+\frac{i(t, 0)\left(1+\alpha i^{*}(0)\right)}{i^{*}(0)(1+\alpha i(t, 0))}\right. \\
& \left.+\ln \frac{i(t, a)\left(1+\alpha i^{*}(a)\right)}{i^{*}(a)(1+\alpha i(t, a))}-\ln \frac{i(t, 0)\left(1+\alpha i^{*}(0)\right)}{i^{*}(0)(1+\alpha i(t, 0))}\right\} \mathrm{d} a \\
& +\int_{0}^{\infty} \beta_{3}(a) S^{*} j^{*}(a)\left\{1-\frac{S(t) j(t, a)}{S^{*} j^{*}(a)}-\frac{S^{*}}{S(t)}+\frac{j(t, 0)}{j^{*}(0)}+\ln \frac{j(t, a)}{j^{*}(a)}-\ln \frac{j(t, 0)}{j^{*}(0)}\right\} \mathrm{d} a \\
& +\int_{0}^{\infty} \beta_{4}(a) V^{*} j^{*}(a)\left\{-1-\frac{V(t) j(t, a)}{V^{*} j^{*}(a)}+\frac{V^{*}}{V(t)}+\frac{j(t, 0)}{j^{*}(0)}\right. \\
& \left.+\ln \frac{j(t, a)}{j^{*}(a)}-\ln \frac{j(t, 0)}{j^{*}(0)}\right\} \mathrm{d} a \\
& +\int_{0}^{\infty} \frac{S^{*} \beta_{1}(a) B_{2}(a)+V^{*} \beta_{2}(a) B_{2}(a)}{1+\alpha i^{*}(a)} \mathrm{d} a \int_{0}^{\infty} \gamma_{1}(a) e^{*}(a)\left\{\frac{e(t, 0)}{e^{*}(0)}-\frac{e(t, a)}{e^{*}(a)}\right. \\
& \left.+\ln \frac{e(t, a)}{e^{*}(a)}-\ln \frac{e(t, 0)}{e^{*}(0)}\right\} \mathrm{d} a \\
& +\int_{0}^{\infty}\left(S^{*} \beta_{3}(a) B_{3}(a)+V^{*} \beta_{4}(a) B_{3}(a)\right) \mathrm{d} a \int_{0}^{\infty} \gamma_{2}(a) e^{*}(a)\left\{\frac{e(t, 0)}{e^{*}(0)}-\frac{e(t, a)}{e^{*}(a)}\right. \\
& \left.+\ln \frac{e(t, a)}{e^{*}(a)}-\ln \frac{e(t, 0)}{e^{*}(0)}\right\} \mathrm{d} a \\
& +\int_{0}^{\infty}\left(S^{*} \beta_{3}(a) B_{3}(a)+V^{*} \beta_{4}(a) B_{3}(a)\right) \mathrm{d} a \int_{0}^{\infty} \xi(a) i^{*}(a)\left\{\frac{i(t, 0)}{i^{*}(0)}-\frac{i(t, a)}{i^{*}(a)}\right. \\
& \left.+\ln \frac{i(t, a)}{i^{*}(a)}-\ln \frac{i(t, 0)}{i^{*}(0)}\right\} \mathrm{d} a \\
& =\mu S^{*}\left(2-\frac{S(t)}{S^{*}}-\frac{S^{*}}{S(t)}\right)+\left(\mu+\rho+\int_{0}^{\infty}\left(\frac{\beta_{2}(a) i^{*}(a)}{1+\alpha i^{*}(a)}+\beta_{4}(a) j^{*}(a)\right) \mathrm{d} a\right) V^{*} \\
& \times\left(3-\frac{V(t)}{V^{*}}-\frac{S^{*}}{S(t)}-\frac{S(t) V^{*}}{S^{*} V(t)}\right)+\eta V^{*}\left(2-\frac{S^{*} V(t)}{S(t) V^{*}}-\frac{S(t) V^{*}}{S^{*} V(t)}\right) \\
& +\int_{0}^{\infty} \frac{\beta_{1}(a) S^{*} i^{*}(a)}{1+\alpha i^{*}(a)}\left\{1-\frac{S^{*}}{S(t)}+\ln \frac{i(t, a)\left(1+\alpha i^{*}(a)\right)}{i^{*}(a)(1+\alpha i(t, a))}\right. \\
& \left.-\ln \frac{i(t, 0)\left(1+\alpha i^{*}(0)\right)}{i^{*}(0)(1+\alpha i(t, 0))}\right\} \mathrm{d} a
\end{aligned}
$$




$$
\begin{aligned}
& +\int_{0}^{\infty} \frac{\beta_{2}(a) V^{*} i^{*}(a)}{1+\alpha i^{*}(a)}\left\{-1+\frac{V^{*}}{V(t)}+\ln \frac{i(t, a)\left(1+\alpha i^{*}(a)\right)}{i^{*}(a)(1+\alpha i(t, a))}\right. \\
& \left.-\ln \frac{i(t, 0)\left(1+\alpha i^{*}(0)\right)}{i^{*}(0)(1+\alpha i(t, 0))}\right\} \mathrm{d} a \\
& +\int_{0}^{\infty} \beta_{3}(a) S^{*} j^{*}(a)\left\{1-\frac{S^{*}}{S(t)}+\ln \frac{j(t, a)}{j^{*}(a)}-\ln \frac{j(t, 0)}{j^{*}(0)}\right\} \mathrm{d} a \\
& +\int_{0}^{\infty} \beta_{4}(a) V^{*} j^{*}(a)\left\{-1+\frac{V^{*}}{V(t)}+\ln \frac{j(t, a)}{j^{*}(a)}-\ln \frac{j(t, 0)}{j^{*}(0)}\right\} \mathrm{d} a \\
& +\int_{0}^{\infty} \frac{S^{*} \beta_{1}(a) B_{2}(a)+V^{*} \beta_{2}(a) B_{2}(a)}{1+\alpha i^{*}(a)} \mathrm{d} a \\
& \times \int_{0}^{\infty} \gamma_{1}(a) e^{*}(a)\left\{\ln \frac{e(t, a)}{e^{*}(a)}-\ln \frac{e(t, 0)}{e^{*}(0)}\right\} \mathrm{d} a \\
& +\int_{0}^{\infty}\left(S^{*} \beta_{3}(a) B_{3}(a)+V^{*} \beta_{4}(a) B_{3}(a)\right) \mathrm{d} a \\
& \times \int_{0}^{\infty} \gamma_{2}(a) e^{*}(a)\left\{\ln \frac{e(t, a)}{e^{*}(a)}-\ln \frac{e(t, 0)}{e^{*}(0)}\right\} \mathrm{d} a \\
& +\int_{0}^{\infty}\left(S^{*} \beta_{3}(a) B_{3}(a)+V^{*} \beta_{4}(a) B_{3}(a)\right) \mathrm{d} a \\
& \times \int_{0}^{\infty} \xi(a) i^{*}(a)\left\{\ln \frac{i(t, a)}{i^{*}(a)}-\ln \frac{i(t, 0)}{i^{*}(0)}\right\} \mathrm{d} a \\
& +\int_{0}^{\infty} \frac{S^{*} \beta_{1}(a) B_{2}(a)+V^{*} \beta_{2}(a) B_{2}(a)}{1+\alpha i^{*}(a)} \mathrm{d} a \int_{0}^{\infty} \gamma_{1}(a) e^{*}(a)\left\{\frac{e(t, 0)}{e^{*}(0)}\right\} \mathrm{d} a \\
& \left.+\int_{0}^{\infty}\left(S^{*} \beta_{3}(a) B_{3}(a)+V^{*} \beta_{4}(a) B_{3}(a)\right) \mathrm{d} a\right) \int_{0}^{\infty} \gamma_{2}(a) e^{*}(a)\left\{\frac{e(t, 0)}{e^{*}(0)}\right\} \mathrm{d} a \\
& +\int_{0}^{\infty}\left(S^{*} \beta_{3}(a) B_{3}(a)+V^{*} \beta_{4}(a) B_{3}(a)\right) \mathrm{d} a \int_{0}^{\infty} \xi(a) i^{*}(a)\left\{\frac{i(t, 0)}{i^{*}(0)}\right\} \mathrm{d} a \\
& -\int_{0}^{\infty} \frac{\beta_{1}(a) S^{*} i^{*}(a)}{1+\alpha i^{*}(a)} \frac{S(t) i(t, a)\left(1+\alpha i^{*}(a)\right)}{S^{*} i^{*}(a)(1+\alpha i(t, a))} \mathrm{d} a \\
& -\int_{0}^{\infty} \frac{\beta_{2} V^{*}(a) i^{*}(a)}{1+\alpha i^{*}(a)} \frac{V(t) i(t, a)\left(1+\alpha i^{*}(a)\right)}{V^{*} i^{*}(a)(1+\alpha i(t, a))} \mathrm{d} a \\
& -\int_{0}^{\infty} \beta_{3}(a) S^{*} j^{*}(a) \frac{S(t) j(t, a)}{S^{*} j^{*}(a)} \mathrm{d} a-\int_{0}^{\infty} \beta_{4}(a) V^{*} j^{*}(a) \frac{V(t) j(t, a)}{V^{*} j^{*}(a)} \mathrm{d} a \\
& -\left(K_{3} S^{*}+K_{5} V^{*}\right) i(t, 0)-\left(K_{4} S^{*}+K_{6} V^{*}\right) j(t, 0) \\
& +\int_{0}^{\infty} \frac{\beta_{1}(a) S^{*}+\beta_{2}(a) V^{*}}{1+\alpha i^{*}(a)} i^{*}(a) \frac{i(t, 0)\left(1+\alpha i^{*}(0)\right)}{i^{*}(0)(1+\alpha i(t, 0))} \mathrm{d} a \\
& +\int_{0}^{\infty}\left(\beta_{3}(a) S^{*}+\beta_{4}(a) V^{*}\right) j^{*}(a) \frac{j(t, 0)}{j^{*}(0)} \mathrm{d} a \text {. }
\end{aligned}
$$

It is easy to see that the last 11 terms of the above equation equal 0 . Then we have

$$
\begin{aligned}
\frac{\mathrm{d} V_{2}}{\mathrm{~d} t} \leq & \mu S^{*}\left(2-\frac{S(t)}{S^{*}}-\frac{S^{*}}{S(t)}\right)+(\mu+\rho) V^{*}\left(3-\frac{V(t)}{V^{*}}-\frac{S^{*}}{S(t)}-\frac{S(t) V^{*}}{S^{*} V(t)}\right) \\
& +\eta V^{*}\left(2-\frac{S^{*} V(t)}{S(t) V^{*}}-\frac{S(t) V^{*}}{S^{*} V(t)}\right)
\end{aligned}
$$




$$
\begin{aligned}
& +\int_{0}^{\infty}\left(\frac{\beta_{2}(a) V^{*} i^{*}(a)}{1+\alpha i^{*}(a)}+\beta_{4}(a) V^{*} j^{*}(a)\right)\left\{3-\frac{V(t)}{V^{*}}-\frac{S^{*}}{S(t)}-\frac{S(t) V^{*}}{S^{*} V(t)}\right\} \mathrm{d} a \\
& +\int_{0}^{\infty} \frac{\beta_{1}(a) S^{*} i^{*}(a)}{1+\alpha i^{*}(a)}\left\{1-\frac{S^{*}}{S(t)}+\ln \frac{i(t, a)\left(1+\alpha i^{*}(a)\right)}{i^{*}(a)(1+\alpha i(t, a))}\right. \\
& \left.-\ln \frac{i(t, 0)\left(1+\alpha i^{*}(0)\right)}{i^{*}(0)(1+\alpha i(t, 0))}\right\} \mathrm{d} a \\
& +\int_{0}^{\infty} \frac{\beta_{2}(a) V^{*} i^{*}(a)}{1+\alpha i^{*}(a)}\left\{-1+\frac{V^{*}}{V(t)}+\ln \frac{i(t, a)\left(1+\alpha i^{*}(a)\right)}{i^{*}(a)(1+\alpha i(t, a))}\right. \\
& \left.-\ln \frac{i(t, 0)\left(1+\alpha i^{*}(0)\right)}{i^{*}(0)(1+\alpha i(t, 0))}\right\} \mathrm{d} a \\
& +\int_{0}^{\infty} \beta_{3}(a) S^{*} j^{*}(a)\left\{1-\frac{S^{*}}{S(t)}+\ln \frac{j(t, a)}{j^{*}(a)}-\ln \frac{j(t, 0)}{j^{*}(0)}\right\} \mathrm{d} a \\
& +\int_{0}^{\infty} \beta_{4}(a) V^{*} j^{*}(a)\left\{-1+\frac{V^{*}}{V(t)}+\ln \frac{j(t, a)}{j^{*}(a)}-\ln \frac{j(t, 0)}{j^{*}(0)}\right\} \mathrm{d} a \\
& +\int_{0}^{\infty} \frac{S^{*} \beta_{1}(a) B_{2}(a)+V^{*} \beta_{2}(a) B_{2}(a)}{1+\alpha i^{*}(a)} \mathrm{d} a \\
& \times \int_{0}^{\infty} \gamma_{1}(a) e^{*}(a)\left\{\ln \frac{e(t, a)}{e^{*}(a)}-\ln \frac{e(t, 0)}{e^{*}(0)}\right\} \mathrm{d} a \\
& +\int_{0}^{\infty}\left(S^{*} \beta_{3}(a) B_{3}(a)+V^{*} \beta_{4}(a) B_{3}(a)\right) \mathrm{d} a \\
& \times \int_{0}^{\infty} \gamma_{2}(a) e^{*}(a)\left\{\ln \frac{e(t, a)}{e^{*}(a)}-\ln \frac{e(t, 0)}{e^{*}(0)}\right\} \mathrm{d} a \\
& +\int_{0}^{\infty}\left(S^{*} \beta_{3}(a) B_{3}(a)+V^{*} \beta_{4}(a) B_{3}(a)\right) \mathrm{d} a \\
& \times \int_{0}^{\infty} \xi(a) i^{*}(a)\left\{\ln \frac{i(t, a)}{i^{*}(a)}-\ln \frac{i(t, 0)}{i^{*}(0)}\right\} \mathrm{d} a .
\end{aligned}
$$

Consequently, we have

$$
\begin{aligned}
\frac{\mathrm{d} V_{2}}{\mathrm{~d} t} \leq & \mu S^{*}\left(2-\frac{S(t)}{S^{*}}-\frac{S^{*}}{S(t)}\right)+(\mu+\rho) V^{*}\left(3-\frac{V(t)}{V^{*}}-\frac{S^{*}}{S(t)}-\frac{S(t) V^{*}}{S^{*} V(t)}\right) \\
& +\eta V^{*}\left(2-\frac{S^{*} V(t)}{S(t) V^{*}}-\frac{S(t) V^{*}}{S^{*} V(t)}\right) \\
& -\int_{0}^{\infty}\left[\frac{\beta_{1}(a) S^{*}+\beta_{2}(a) V^{*}}{1+\alpha i^{*}(a)} i^{*}(a)+\left(\beta_{3}(a) S^{*}+\beta_{4}(a) V^{*}\right) j^{*}(a)\right] g\left(\frac{S^{*}}{S(t)}\right) \mathrm{d} a \\
& -\int_{0}^{\infty} \frac{S^{*} \beta_{1}(a) B_{2}(a)+V^{*} \beta_{2}(a) B_{2}(a)}{1+\alpha i^{*}(a)} \mathrm{d} a \int_{0}^{\infty} \gamma_{1}(a) e^{*}(a) g\left(\frac{e(t, a) i^{*}(0)}{e^{*}(a) i(t, 0)}\right) \mathrm{d} a \\
& -\int_{0}^{\infty}\left(S^{*} \beta_{3}(a) B_{3}(a)+V^{*} \beta_{4}(a) B_{3}(a)\right) \mathrm{d} a \int_{0}^{\infty} \gamma_{2}(a) e^{*}(a) g\left(\frac{e(t, a) j^{*}(0)}{e^{*}(a) j(t, 0)}\right) \mathrm{d} a \\
& -\int_{0}^{\infty}\left(S^{*} \beta_{3}(a) B_{3}(a)+V^{*} \beta_{4}(a) B_{3}(a)\right) \mathrm{d} a \int_{0}^{\infty} \xi(a) i^{*}(a) g\left(\frac{i(t, a) j^{*}(0)}{i^{*}(a) j(t, 0)}\right) \mathrm{d} a \\
& -\int_{0}^{\infty} \frac{\beta_{1}(a) S^{*} i^{*}(a)}{1+\alpha i^{*}(a)} g\left(\frac{S(t) i(t, a) e^{*}(0)\left(1+\alpha i^{*}(a)\right)}{S^{*} i^{*}(a) e(t, 0)(1+\alpha i(t, a))}\right) \mathrm{d} a \\
& -\int_{0}^{\infty} \frac{\beta_{2}(a) V^{*} i^{*}(a)}{1+\alpha i^{*}(a)} g\left(\frac{V(t) i(t, a) e^{*}(0)\left(1+\alpha i^{*}(a)\right)}{V^{*} i^{*}(a) e(t, 0)(1+\alpha i(t, a))}\right) \mathrm{d} a
\end{aligned}
$$




$$
\begin{aligned}
& -\int_{0}^{\infty} \beta_{3}(a) S^{*} j^{*}(a) g\left(\frac{S(t) j(t, a) e^{*}(0)}{S^{*} j^{*}(a) e(t, 0)}\right) \mathrm{d} a \\
& -\int_{0}^{\infty} \beta_{4}(a) V^{*} j^{*}(a) g\left(\frac{V(t) j(t, a) e^{*}(0)}{V^{*} j^{*}(a) e(t, 0)}\right) \mathrm{d} a \\
& -\int_{0}^{\infty} \frac{\beta_{2}(a) V^{*} i^{*}(a)}{1+\alpha i^{*}(a)} g\left(\frac{S(t) V^{*}}{S^{*} V(t)}\right) \mathrm{d} a \\
& -\int_{0}^{\infty} \beta_{4}(a) V^{*} j^{*}(a) g\left(\frac{S(t) V^{*}}{S^{*} V(t)}\right) \mathrm{d} a .
\end{aligned}
$$

Hence, $\mathrm{d} V_{2} / \mathrm{d} t \leq 0$ holds. Furthermore, the strict equality holds if and only if $S=S^{*}, V=$ $V^{*}, e(t, a)=e^{*}(a), i(t, a)=i^{*}(a), j(t, a)=j^{*}(a)$. Thus, $M^{*}=\left\{E^{*}\right\} \subset \Omega$ is the largest invariant subset of $\mathrm{d} V_{2} / \mathrm{d} t=0$, and by the Lyapunov-LaSalle invariance principle, when $R_{0}>1$, the steady state $E^{*}$ is globally asymptotically stable. This completes the proof.

\section{Discussion}

An age-structured HBV model with saturating incidence has been proposed here to incorporate patients with acute hepatitis B and chronic hepatitis B. By mathematical analysis, the dynamic behavior of system (1.5) was shown to be determined completely by the basic reproduction number $R_{0}$ : disease free steady state $E_{0}$ is locally and globally asymptotically stable if $R_{0}<1$; endemic steady state $E^{*}$ is locally and globally asymptotically stable if $R_{0}>1$. To place the model on more sound biological grounds, we considered the fact that the vaccines may lose efficacy and the infection may reach a saturating state. Next, we will focus on the numerical simulations of such a complex partial differential equations (PDEs) model.

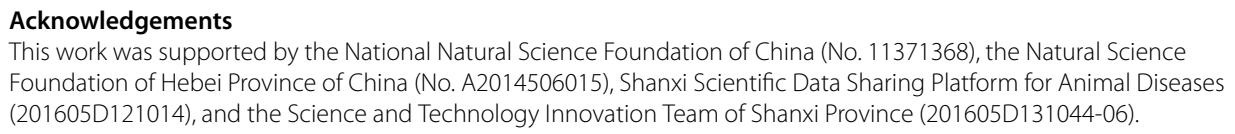

The authors declare that they have no competing interests.

\section{Authors' contributions}

$Y L$ and RX have contributed equally to the writing of this paper except for Sect. 6. JL made major contribution to the revised paper and Sect. 6 . All authors read and approved the final manuscript.

\section{Author details}

${ }^{1}$ Institute of Applied Mathematics, Army Engineering University, Shijiazhuang, P.R. China. ${ }^{2}$ Complex Systems Research Center, Shanxi University, Taiyuan, P.R. China. ${ }^{3}$ Shanxi Key Laboratory of Mathematical Techniques and Big Data Analysis on Disease Control and Prevention, Shanxi University, Taiyuan, P.R. China.

\section{Publisher's Note}

Springer Nature remains neutral with regard to jurisdictional claims in published maps and institutional affiliations.

Received: 19 December 2017 Accepted: 3 May 2018 Published online: 07 June 2018

\section{References}

1. Liu, P.: Dynamical analysis of HBV dynamic models with immune response. Master dissertation (2013)

2. Li, Y., Xu, R.: The stability analysis of an epidemic model with age-structure in the exposed and infectious classes. Commun. Math. Biol. Neurosci. 2017, Article ID 12 (2017)

3. Korobeinikov, A., Maini, P.K.: A Lyapunov function and global properties for SIR and SEIR epidemiological models with nonlinear incidence. Math. Biosci. Eng. 1,57-60 (2004)

4. Li, G., Jin, Z.: Global staility of a SEIR epidemic model with infectious force in latent, infected and immune period. Chaos Solitons Fractals 25, 1177-1184 (2005)

5. Li, M., Graef, J.R., Wang, L., Karsai, J.: Global dynamics of a SEIR model with varying total population size. Math. Biosci. 160, 191-213 (1999) 
6. Li, M., Muldowney, J.S.: Global stability for the SEIR model in epidemiology. Math. Biosci. 12, 155-164 (1995)

7. Zhang, J., Ma, Z.: Global dynamics of an SEIR epidemic model with saturating contact rate. Math. Biosci. 185, 15-32 (2003)

8. McCluskey, C.C.: Global stability for an SEl epidemiological model with continuous age-structure in the exposed and infectious classes. Math. Biosci. Eng. 9(4), 819-841 (2012)

9. Wei, H., Jiang, Y., Song, X., Su, G., Qiu, S.: Global attractivity and permanence of a SVEIR epidemic model with pulse vaccination and time delay. J. Comput. Appl. Math. 229, 302-312 (2009)

10. Li, J., Yang, Y., Zhou, Y.: Global stability of an epidemic model with latent stage and vaccination. Nonlinear Anal., Real World Appl. 12, 2163-2173 (2011)

11. Liu, X., Takeuchi, Y., Iwami, S.: SVIR epidemic models with vaccination strategies. J. Theor. Biol. 253, 1-11 (2008)

12. Xiao, Y., Tang, S.: Dynamics of infection with nonlinear incidence in a simple vaccination model. Nonlinear Anal., Real World Appl. 11, 4154-4163 (2010)

13. Alexander, M.E., Bowman, C., Moghadas, S.M., Summers, R., Gumel, A.B., Sahai, B.M.: A vaccination model for transmission dynamics of influenza. SIAM J. Appl. Dyn. Syst. 3, 503-524 (2004)

14. Li, J., Ma, Z., Zhou, Y.: Global analysis of SIS epidemic model with a simple vaccination and multiple endemic equilibria. Acta Math. Sci. 26, 83-93 (2006)

15. Moghadas, S.M., Gumel, A.B.: A mathematical study of a model for childhood diseases with non-permanent immunity. J. Comput. Appl. Math. 157, 347-363 (2003)

16. McCluskey, C.C.: Global stability for an SEl model of infectious disease with age structure and immigration of infected. Math. Biosci. Eng. 13(2), 381-400 (2016)

17. Magal, P., McCluskey, C., Webb, G.: Lyapunov functional and global asymptotic stability for an infection-age model. Appl. Anal. 89(7), 1109-1140 (2010)

18. Brauer, F., Shuai, Z., van den Driessche, P.: Dynamics of an age-of-infection cholera model. Math. Biosci. Eng. 10(5-6), 1335-1349 (2013)

19. Huang, G., Liu, X., Takeuchi, Y.: Lyapunov functions and global stability for age-structured HIV infection model. SIAM J. Appl. Math. 72(1), 25-38 (2012)

20. Wang, J., Zhang, R., Kuniya, T.: Mathematical analysis for an age-structured HIV infection model with saturation infection rate. Electron. J. Differ. Equ. 2015, 33 (2015)

21. Wang, J., Zhang, R., Kuniya, T.: A note on dynamics of an age-of-infection cholera model. Math. Biosci. Eng. 13(1), 227-247 (2016)

22. Wang, J., Zhang, R., Kuniya, T.: The dynamics of an SVIR epidemiological model with infection age. IMA J. Appl. Math. $81,321-343(2016)$

23. Wang, J., Guo, M., Liu, S.: SVIR epidemic model with age structure in susceptibility, vaccination effects and relapse IMA J. Appl. Math. 82, 945-970 (2017)

24. Hale, J.K.: Functional Diffferential Equations. Springer, Berlin (1971)

25. Hale, J.K., Waltman, P.: Persistence in infinite-dimensional systems. SIAM J. Math. Anal. 20(2), 388-395 (1989)

26. Liu, L., Wang, J., Liu, X.: Global stability of an SEIR epidemic model with age-dependent latency and relapse. Nonlinear Anal., Real World Appl. 24, 18-35 (2015)

27. Smith, H.L., Thieme, H.R.: Dynamical Systems and Population Persistence. Am. Math. Soc., Providence (2011)

28. Wang, J., Zhang, R., Kuniya, T.: The stability analysis of an SVEIR model with continuous age-structure in the exposed and infectious classes. J. Biol. Dyn. 9(1), 73-101 (2015)

\section{Submit your manuscript to a SpringerOpen ${ }^{\circ}$ journal and benefit from:}

- Convenient online submission

- Rigorous peer review

- Open access: articles freely available online

- High visibility within the field

- Retaining the copyright to your article

Submit your next manuscript at $\boldsymbol{\nabla}$ springeropen.com 